\title{
RH-CATALYZED ENANTIOSELECTIVE HYDROGENATION OF VINYL BORONATES FOR THE CONSTRUCTION OF SECONDARY BORONIC ESTERS.
}

\author{
Wesley J. Moran and James P. Morken* \\ Department of Chemistry, Venable and Kenan Laboratories, The University of North Carolina, Chapel Hill, \\ North Carolina 27599-3290
}

Supporting Information

General. ${ }^{1} \mathrm{H}$ NMR spectra were recorded on Bruker DRX 300 or $400 \mathrm{MHz}$ spectrometers. Chemical shifts are reported in ppm from tetramethylsilane with the solvent resonance as the internal standard (CDCl3: $7.24 \mathrm{ppm})$. Data are reported as follows: chemical shift, integration, multiplicity $(\mathrm{s}=$ singlet, $\mathrm{d}=$ doublet, $\mathrm{t}=$ triplet, $\mathrm{q}=$ quartet, $\mathrm{br}=$ broad, $\mathrm{m}=$ multiplet), coupling constants $(\mathrm{Hz})$ and assignment. ${ }^{13} \mathrm{C}$ NMR was recorded on a Bruker $400 \mathrm{MHz}(100 \mathrm{MHz})$ spectrometer with complete proton decoupling. Chemical shifts are reported in $\mathrm{ppm}$ from tetramethylsilane with the solvent as the internal standard $\left(\mathrm{CDCl}_{3}: 77.0 \mathrm{ppm}\right)$. Mass spectrometry $(\mathrm{m} / \mathrm{z})$ was performed on either a Bruker BioTOF II or a Micromass Quattro II, operating in ESI or APCI mode, with only molecular ions reported. Infrared (IR) spectra were obtained on an ASI ReactIR 1000 or a Nicolet 560 Magna-IR, umax in $\mathrm{cm}^{-1}$. Bands are characterized as broad (br), strong (s), medium (m) and weak (w). Flash chromatography was performed on silica gel $\left(\mathrm{SiO}_{2}, 230 \mathrm{X} 450\right.$ mesh) purchased from Sorbent Technologies, Inc. Thin layer chromatography (TLC) was performed on aluminium backed plates pre-coated with silica $(0.2$ mm, Merck DC-alufolien Kieselgel 60). Visualization was achieved using basic potassium permanganate solution, followed by heating. Analytical gas-liquid chromatography (GLC) was performed on a HewlettPackard 6890 Series chromatography equipped with a CTC Analysis Combi Pal autosampler by Leap Technologies (Carrboro, NC), a split mode capillary injection system, a flame ionization detector and a Supelco $\beta$-dex 120 column with helium as the carrier gas. All reactions were conducted in oven and flame dried glassware under an inert atmosphere of nitrogen unless otherwise stated. Walphos 1, $(R)-1-\left[(R)-2-\left(2^{\prime}-\right.\right.$ diphenylphosphinophenyl)ferrocenyl]ethyldi(bis-3,5-trifluoromethylphenyl)phosphine, was kindly donated by Sovias AG. Bis(norbornadiene) rhodium(I) tetrafluoroborate was purchased from Acros Organics. All other reagents were purchased from Aldrich Chemical Companies and used directly. Hydrogenations were performed in a stainless steel high-pressure vessel from Parr Instrument Company. Enantiomeric excesses of the boronate compounds were determined by chiral GC analysis after oxidation of the carbon boron bond followed by acetate derivatization. Absolute configuration of products determined by comparison of GC data of commercial nonracemic chiral alcohols.

A note about NMR spectra: Due to the boron quadrupole, carbons directly attached to this element are often not detected in ${ }^{13} \mathrm{C}$ spectra. See Wrackmeyer, B. Prog. In NMR Spectroscopy, 1979, 12, 227. In some cases, the ${ }^{2} \mathrm{~J}$ and ${ }^{3} \mathrm{~J}{ }^{11} \mathrm{~B} /{ }^{1} \mathrm{H}$ and ${ }^{10} \mathrm{~B} /{ }^{1} \mathrm{H}$ coupling makes determination of some ${ }^{1} \mathrm{H} /{ }^{1} \mathrm{H}$ coupling constants difficult.

\section{Representative Procedure for Preparation of Vinyl Boronic Acid Pinacol Esters: Synthesis of 2-(1-Hexyl-} vinyl)-4,4,5,5-tetramethyl-[1,3,2]dioxaborolane.

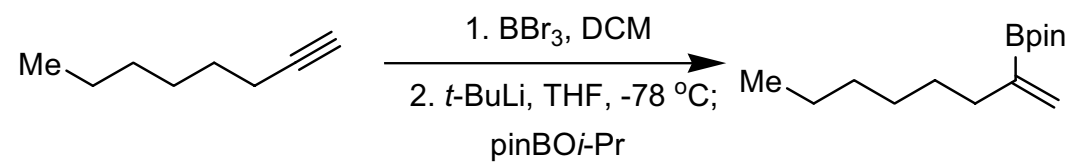

1-Octyne $(1.3 \mathrm{ml}, 9.1 \mathrm{mmol}, 1$ equiv) was added dropwise to a $1 \mathrm{M}$ solution of boron tribromide $(4.5 \mathrm{ml}, 4.5$ mmol, 0.5 equiv) at $-78^{\circ} \mathrm{C}$. The resulting solution was allowed to warm to room temperature over 3 h. Glacial acetic acid $(9 \mathrm{ml})$ was added to the mixture and stirred for $1 \mathrm{~h}$. This mixture was quenched with water, extracted with DCM and dried over MgSO4. Filtration, followed by (careful) concentration in vacuo and passage through a short silica column (hexanes) provided the desired vinyl bromide sufficiently clean to continue on to the next step. The vinyl bromide was dissolved in THF $(15 \mathrm{ml})$ and cooled to $-78^{\circ} \mathrm{C}$. $\mathrm{t}-\mathrm{BuLi}$ $\left(1.5 \mathrm{M}, 12 \mathrm{ml}, 18 \mathrm{mmol}, 2\right.$ equiv) was added dropwise, and the resulting mixture stirred for $0.5-1 \mathrm{~h}$ at $-78^{\circ} \mathrm{C}$. 2-Isopropoxy-4,4,5,5-tetramethyl-[1,3,2]dioxaborolane $(2.8 \mathrm{ml}, 14 \mathrm{mmol}, 1.5$ equiv) dissolved in THF (10 ml) was added dropwise via cannula and the mixture allowed to warm to room temperature over $3 \mathrm{~h} .1 \mathrm{M} \mathrm{HCl}$ solution $(20 \mathrm{ml})$ was added and the mixture was stirred for $0.5 \mathrm{~h}$. Extraction with DCM, followed by drying 
Page S- 2

over $\mathrm{MgSO}$, filtration and concentration in vacuo provided the crude oil. Purification by flash chromatography (20:1 hexanes / EtOAc) furnished a colorless oil (1.8 g, 83\%). IR (neat): 2975 (s), 2861 (s), $1721(\mathrm{~s}), 1615(\mathrm{~s}), 1142(\mathrm{br}) \mathrm{cm}^{-1} .{ }^{1} \mathrm{H}$ NMR $\left(400 \mathrm{MHz}, \mathrm{CDCl}_{3}\right): \delta 0.83(3 \mathrm{H}, \mathrm{t}, \mathrm{J}=6.4 \mathrm{~Hz}), 1.15-1.42(20 \mathrm{H}, \mathrm{m})$, $2.11(2 \mathrm{H}, \mathrm{t}, \mathrm{J}=6.4 \mathrm{~Hz}), 5.57(1 \mathrm{H}, \mathrm{br}), 5.72(1 \mathrm{H}, \mathrm{br}) .{ }^{13} \mathrm{C} \mathrm{NMR}\left(100.6 \mathrm{MHz}, \mathrm{CDCl}_{3}\right): \delta 14.5,23.0,25.1,29.4$, $29.6,32.2,35.8,83.7,129.1$.

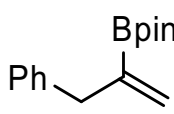

2-(1-benzyl-vinyl)-4,4,5,5-tetramethyl-[1,3,2]dioxaborolane. IR (neat): 2981 (s), 2931 (s), 1947 (w), 1887 (w), 1713 (s), 1615 (s) cm ${ }^{-1}$. ${ }^{1} \mathrm{H}$ NMR (400 MHz, CDCl $): \delta 1.21$ (12H, s), 3.47 $(2 \mathrm{H}, \mathrm{s}), 5.51(1 \mathrm{H}, \mathrm{br}), 5.82(1 \mathrm{H}, \mathrm{br}), 7.13-7.25(5 \mathrm{H}, \mathrm{m}) .{ }^{13} \mathrm{C} \mathrm{NMR}\left(100.6 \mathrm{MHz}, \mathrm{CDCl}_{3}\right): \delta 24.8$, $41.6,83.7,125.9,128.3,129.4,130.1,140.9$.

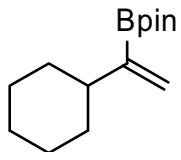

2-(1-cyclohexyl-vinyl)-4,4,5,5-tetramethyl-[1,3,2]dioxaborolane. IR (neat): 2979 (s), 2925 (s), 2852 (s), $1611(\mathrm{~m}), 1306$ (s), $1144(\mathrm{~s}) \mathrm{cm}^{-1}$. ${ }^{1} \mathrm{H}$ NMR (400 MHz, $\left.\mathrm{CDCl}_{3}\right): \delta 1.10-1.70(22 \mathrm{H}$, $\mathrm{m}), 2.00-2.16(1 \mathrm{H}, \mathrm{m}), 5.55(1 \mathrm{H}, \mathrm{br}), 5.70(1 \mathrm{H}, \mathrm{br}) .{ }^{13} \mathrm{C} \mathrm{NMR}\left(100.6 \mathrm{MHz}, \mathrm{CDCl}_{3}\right): \delta 14.1$, $24.9,26.9,32.7,43.0,83.4,126.1$.

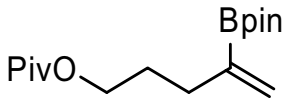

2,2-dimethyl-propionic acid 4-(4,4,5,5-tetramethyl-[1,3,2]dioxaborolan-2-yl)-pent-4enyl ester. IR (neat): 3025 (s), 2900 (s), 1727 (s), 1484 (m) $\mathrm{cm}^{-1}$. ${ }^{1} \mathrm{H}$ NMR (300 MHz, $\left.\mathrm{CDCl}_{3}\right): \delta 1.20(9 \mathrm{H}, \mathrm{s}), 1.26(12 \mathrm{H}, \mathrm{s}), 1.67-1.80(2 \mathrm{H}, \mathrm{m}), 2.22(2 \mathrm{H}, \mathrm{t}, \mathrm{J}=7.7 \mathrm{~Hz}), 4.04$ $(2 \mathrm{H}, \mathrm{t}, \mathrm{J}=6.6 \mathrm{~Hz}), 5.62(1 \mathrm{H}, \mathrm{br}), 5.80(1 \mathrm{H}, \mathrm{br}) .{ }^{13} \mathrm{C} \mathrm{NMR}\left(75.5 \mathrm{MHz}, \mathrm{CDCl}_{3}\right): \delta 22.9$, 25.0, 27.4, 28.4, 39.0, 64.1, 83.6, 130.1, 178.3.

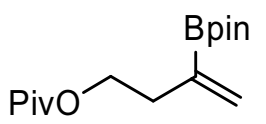

2,2-dimethyl-propionic acid 3-(4,4,5,5-tetramethyl-[1,3,2] dioxaborolan-2-yl)-but-3enyl ester. IR (neat): 3025 (s), 2914 (s), 1756 (s), 1360 (s) cm-1. ${ }^{1} \mathrm{H}$ NMR (400 MHz, $\left.\mathrm{CDCl}_{3}\right): \delta 1.15(9 \mathrm{H}, \mathrm{s}), 1.24(12 \mathrm{H}, \mathrm{s}), 2.45(2 \mathrm{H}, \mathrm{t}, \mathrm{J}=6.8 \mathrm{~Hz}), 4.13(2 \mathrm{H}, \mathrm{t}, \mathrm{J}=7.0 \mathrm{~Hz}), 5.66$ (1H, br), $5.84(1 \mathrm{H}, \mathrm{br}) .{ }^{13} \mathrm{C} \mathrm{NMR}\left(100.6 \mathrm{MHz} \mathrm{CDCl}_{3}\right): \delta 25.2,27.6,35.0,39.1,63.8,83.9$, $132.3,178.9$.

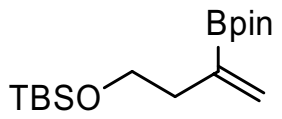

2-\{1-[2-(tert-butyl-dimethyl-silanyloxy)-ethyl]-vinyl $\}-4,4,5,5$-tetramethyl-[1,3,2]dioxa borolane. IR (neat): 2933 (s), 1723 (s), 1382 (s) $\mathrm{cm}^{-1} .{ }^{1} \mathrm{H}$ NMR $\left(400 \mathrm{MHz}, \mathrm{CDCl}_{3}\right): \delta$ $0.01(6 \mathrm{H}, \mathrm{s}), 0.87(9 \mathrm{H}, \mathrm{s}), 1.26(12 \mathrm{H}, \mathrm{s}), 2.37(2 \mathrm{H}, \mathrm{t}, \mathrm{J}=7.2 \mathrm{~Hz}), 3.65(2 \mathrm{H}, \mathrm{t}, \mathrm{J}=7.2 \mathrm{~Hz})$, $5.65(1 \mathrm{H}, \mathrm{br}), 5.82(1 \mathrm{H}, \mathrm{br}) .{ }^{13} \mathrm{C}$ NMR $\left(100.5 \mathrm{MHz}, \mathrm{CDCl}_{3}\right): \delta-5.2,18.4,24.7,26.0$, $39.1,63.1,83.3,131.8$.

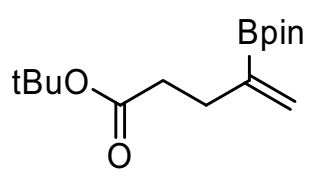

2-\{1-[2-(tert-butyl-dimethyl-silanyloxy)-ethyl]-vinyl\}-4,4,5,5-tetramethyl-[1,3,2]dioxaborolane. IR (neat): 2979 (s), 2931 (m), 1733(s), 1370 (s), 1312 (s), 1142 (s) cm cm $^{-1}$. ${ }^{1} \mathrm{H}$ NMR $\left(300 \mathrm{MHz}, \mathrm{CDCl}_{3}\right): \delta 1.26(12 \mathrm{H}, \mathrm{s}), 1.44(9 \mathrm{H}, \mathrm{s}), 2.30-2.43(2 \mathrm{H}, \mathrm{m}), 5.61(1 \mathrm{H}$, br), $5.76\left(1 \mathrm{H}\right.$, br). ${ }^{13} \mathrm{C} \mathrm{NMR}\left(75.5 \mathrm{MHz}, \mathrm{CDCl}_{3}\right): \delta 25.0,28.4,31.1,35.3,80.2,83.6$, $130.0,173.0$.

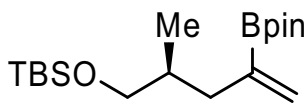

t-Butyldimethyl(2-methyl-4-(4,4,5,5-tetramethyl-1,3,2-dioxaborolan-2-yl)pent-4enyloxy)silane. IR (neat): 2981 (s), 2929 (s), 2858 (s), 1615 (m), 1472 (s), 1372 (s), $1146(\mathrm{~s}), 1092(\mathrm{~s}) \mathrm{cm}^{-1}$. ${ }^{1} \mathrm{H}$ NMR $\left(400 \mathrm{MHz}, \mathrm{CDCl}_{3}\right): \delta 0.00(6 \mathrm{H}, \mathrm{s}), 0.81(3 \mathrm{H}, \mathrm{d}, \mathrm{J}=$ $8.0 \mathrm{~Hz}), 0.87(9 \mathrm{H}, \mathrm{s}), 1.23(12 \mathrm{H}, \mathrm{s}), 1.70-1.90(2 \mathrm{H}, \mathrm{m}), 2.43-2.52(1 \mathrm{H}, \mathrm{dd}, \mathrm{J}=12,8.0$ $\mathrm{Hz}), 3.30(1 \mathrm{H}, \mathrm{dd}, \mathrm{J}=8.0,8.0 \mathrm{~Hz}), 3.45(1 \mathrm{H}, \mathrm{dd}, \mathrm{J}=12,8.0 \mathrm{~Hz}), 5.55(1 \mathrm{H}, \mathrm{br}), 5.77(1 \mathrm{H}, \mathrm{br}) .{ }^{13} \mathrm{C} \mathrm{NMR}(100.6$ $\left.\mathrm{MHz}, \mathrm{CDCl}_{3}\right): \delta-5.3,16.4,18.1,24.5,25.8,35.4,39.0,67.9,83.1,130.2$. 
Synthesis of 4,4,5,5-tetramethyl-2-(1-(R)-methyl-heptyl)-[1,3,2]dioxaborolane. ${ }^{1}$
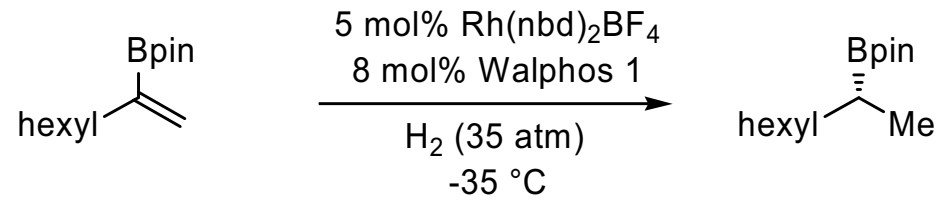

Reaction in 1,2-dichloroethane: A vial was charged with bis(norbornadiene)rhodium(I) tetrafluoroborate (3.9 $\mathrm{mg}, 0.010 \mathrm{mmol}, 0.05$ equiv), Walphos 1 (16 mg, $0.017 \mathrm{mmol}, 0.08$ equiv) and 1,2-dichloroethane (1 ml) in a dry-box and stirred at room temperature for 1 minute. 2-(1-Hexyl-vinyl)-4,4,5,5-tetramethyl$[1,3,2]$ dioxaborolane $(50 \mathrm{mg}, 0.21 \mathrm{mmol}, 1$ equiv) was added to the catalyst solution and the vial was capped and removed from the dry-box. The cap was removed and the vial placed in a Parr Instruments pressure vessel. The vessel was partly submerged in a cryo-bath and cooled to approximately $-35{ }^{\circ} \mathrm{C}$, then purged with $\mathrm{H}_{2}$. The vessel was charged with 30 bar $\mathrm{H}_{2}$ and then depressurized. The vessel was re-charged with 30 bar $\mathrm{H}_{2}$ and left stirring for $15 \mathrm{~h}$. The pressure was released and the vessel removed from the cryo-bath. The vial was removed from the pressure vessel, and the volatiles were removed in vacuo. The resulting residue was purified by flash chromatography (20:1 hexanes/ethyl acetate) to furnish a colorless oil (50 mg, >95\% yield). IR (neat): 2957 (m), 2927 (s), 2855 (m), 1464 (m), 1371 (s), 1313 (s), 1145 (s) cm ${ }^{-1} .{ }^{1} \mathrm{H} \mathrm{NMR}\left(300 \mathrm{MHz}, \mathrm{CDCl}_{3}\right): \delta 0.83$ (3H, t, $\mathrm{J}=6.6 \mathrm{~Hz}), 0.94\left(3 \mathrm{H}\right.$, br s), $1.1-1.5(27 \mathrm{H}, \mathrm{m}) .{ }^{13} \mathrm{C}$ NMR $\left(75.5 \mathrm{MHz}, \mathrm{CDCl}_{3}\right): \delta 14.0,15.4,22.6,24.7(2 \mathrm{C})$, $28.9,29.5,31.8,33.2,82.7$. GC of oxidation/acylation adduct: $85 \%$ ee; $100{ }^{\circ} \mathrm{C}, 25 \mathrm{psi}, \mathrm{t}_{\mathrm{s}}=8.8 \mathrm{~min}, \mathrm{t}_{\mathrm{R}}=10.0 \mathrm{~min}$.

Reaction in toluene: A vial was charged with bis(norbornadiene)rhodium(I) tetrafluoroborate $(3.9 \mathrm{mg}, 0.010$ mmol, 0.05 equiv), Walphos 1 (16 mg, $0.017 \mathrm{mmol}, 0.08$ equiv) and toluene $(1 \mathrm{ml})$ in a dry-box and stirred at room temperature for 60 minutes. 2-(1-Hexyl-vinyl)-4,4,5,5-tetramethyl-[1,3,2]dioxaborolane $(50 \mathrm{mg}, 0.21$ mmol, 1 equiv) was added to the catalyst solution and the vial was capped and removed from the dry-box. The cap was removed and the vial placed in a Parr Instruments pressure vessel. The vessel was partly submerged in a cryo-bath and cooled to approx. $-45^{\circ} \mathrm{C}$, then purged with $\mathrm{H}_{2}$. The vessel was charged with 30 bar $\mathrm{H}_{2}$ and then depressurized. The vessel was re-charged with $30 \mathrm{bar} \mathrm{H}_{2}$ and left stirring for $40 \mathrm{~h}$. The pressure was released and the vessel removed from the cryo-bath. The vial was taken from the pressure vessel, and the volatiles were removed in vacuo. The resulting residue was purified by flash chromatography (20:1 hexanes/ethyl acetate) to furnish a colorless oil (50 mg, >99\% yield, $81 \%$ ee; GLC as above ).

$G C$ conditions: $100^{\circ} \mathrm{C}, 25 \mathrm{psi}, t_{S}=8.8 \mathrm{~min}, t_{R}=10.0 \mathrm{~min} ;$ oxidation/acylation product .

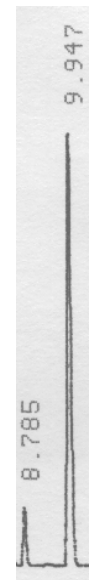

Product

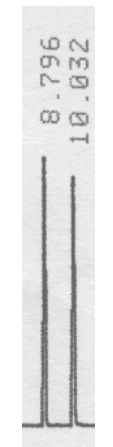

Racemate

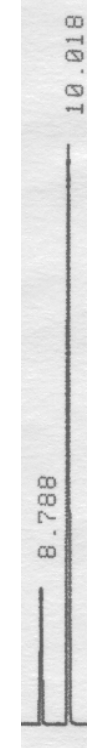

Product + Racemate

(1) Absolute configuration determined by GLC comparison to protected commercially available (R)-(-)-2-octanol. 


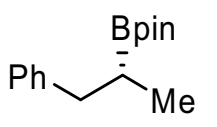

4,4,5,5-tetramethyl-2-(1-(R)-methyl-2-phenyl-ethyl)-[1,3,2] dioxaborolane ${ }^{2}$ : IR (neat): 3062 (m), 3027 (m), 2979 (s), 2931 (s), 1457 (s), 1144 (s) cm ${ }^{-1} .{ }^{1} \mathrm{H}$ NMR (300 MHz, $\left.\mathrm{CDCl}_{3}\right)$ : $\delta 0.94(3 \mathrm{H}, \mathrm{d}, \mathrm{J}=7.5 \mathrm{~Hz}), 1.17(12 \mathrm{H}, \mathrm{s}), 1.35(1 \mathrm{H}, \mathrm{m}), 2.54(1 \mathrm{H}, \mathrm{dd}, \mathrm{J}=13.8,8.2 \mathrm{~Hz}), 2.78$ $(1 \mathrm{H}, \mathrm{dd}, \mathrm{J}=13.5,7.5 \mathrm{~Hz}), 7.16-7.28(5 \mathrm{H}, \mathrm{m}) .{ }^{13} \mathrm{C} \mathrm{NMR}\left(75.5 \mathrm{MHz}, \mathrm{CDCl}_{3}\right): \delta 15.2,24.7(2 \mathrm{C})$, $39.0,83.0,125.5,128.0,128.0,142.3$.

GC conditions: $120^{\circ} \mathrm{C}, 25 \mathrm{psi}, t_{S}=17.0 \mathrm{~min}, t_{R}=17.9 \mathrm{~min}$; oxidation/acylation product .

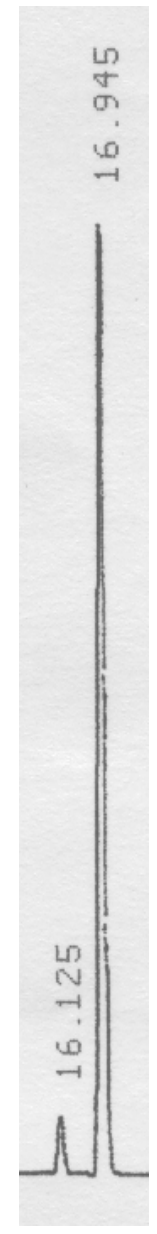

Product

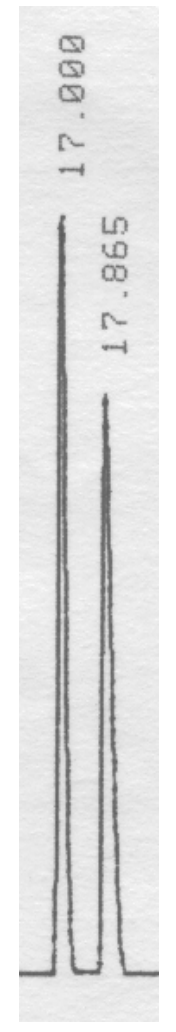

Racemate

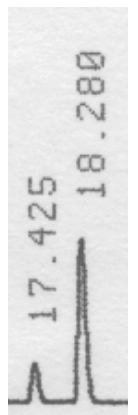

Product + Racemate

(2) Absolute configuration determined by GLC comparison to protected commercially available (R)-(-)-1-phenyl-2-propanol. 
Page S- 5

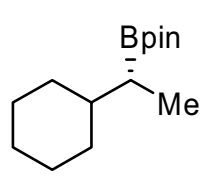

2-(R)-(1-cyclohexyl-ethyl)-4,4,5,5-tetramethyl-[1,3,2] dioxaborolane ${ }^{3}$ : IR (neat): 2979 (s), 2925 (s), 2852 (s), 1449 (s), 1380 (s), $1146(\mathrm{~s}) \mathrm{cm}^{-1}$. ${ }^{1} \mathrm{H}$ NMR (300 MHz, $\left.\mathrm{CDCl}_{3}\right): \delta 0.9-1.40$ $(19 \mathrm{H}, \mathrm{s}), 1.53-1.74(6 \mathrm{H}, \mathrm{m}), 1.95-2.05(2 \mathrm{H}, \mathrm{m}) .{ }^{13} \mathrm{C} \mathrm{NMR}\left(100.5 \mathrm{MHz}, \mathrm{CDCl}_{3}\right): \delta 13.5,25.8$, (2C), $27.7,32.8$ (2C), 33.7 (2C), 41.5, 83.5. MS (A.P.C.I.+) m/z calc'd for $\mathrm{C}_{14} \mathrm{H}_{28} \mathrm{BO}_{2}$ : 239.2. Found: 239.1 .

GC conditions: $100{ }^{\circ} \mathrm{C}, 25 \mathrm{psi}, t_{S}=16.5 \mathrm{~min}, t_{R}=19.2 \mathrm{~min}$; oxidation/acylation product .

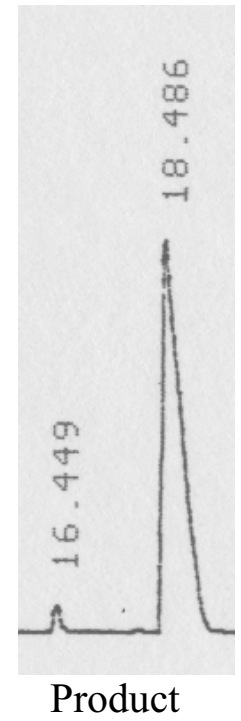

Product

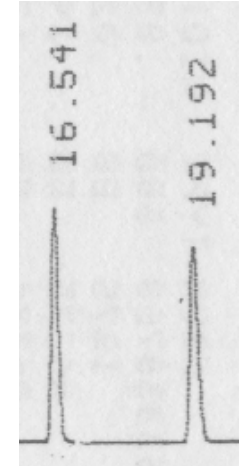

Racemate

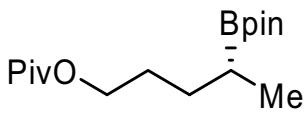

2,2-dimethyl-propionic acid 4-(R)-(4,4,5,5-tetramethyl-[1,3,2]dioxaborolan-2-yl)pentyl ester. IR (neat): 2960 (s), 1723 (s), 1463 (s), $1382(\mathrm{~s}) \mathrm{cm}^{-1} .{ }^{1} \mathrm{H}$ NMR $(400 \mathrm{MHz}$, $\left.\mathrm{CDCl}_{3}\right): \delta$ 0.90-1.06 $(4 \mathrm{H}, \mathrm{m}), 1.15(9 \mathrm{H}, \mathrm{s}), 1.19(12 \mathrm{H}, \mathrm{s}), 1.23-1.37(1 \mathrm{H}, \mathrm{m}), 1.42-1.53$ $(1 \mathrm{H}, \mathrm{m}), 1.54-1.65(2 \mathrm{H}, \mathrm{m}), 4.00(2 \mathrm{H}, \mathrm{t}, \mathrm{J}=6.6 \mathrm{~Hz}) .{ }^{13} \mathrm{C} \mathrm{NMR}\left(100.5 \mathrm{MHz}, \mathrm{CDCl}_{3}\right)$ : $\delta 15.4,24.7,27.2,28.0,29.4,38.7,64.6,82.9,178.8$. MS (E.S.I.) $\mathrm{m} / \mathrm{z}$ calc'd for $\mathrm{C}_{16} \mathrm{H}_{32} \mathrm{BO}_{4}$ : 299.2. Found: 299.2.

GC conditions: $130{ }^{\circ} \mathrm{C}, 25 \mathrm{psi}, t_{S}=13.5 \mathrm{~min}, t_{R}=14.8 \mathrm{~min} ;$ oxidation/acylation product .

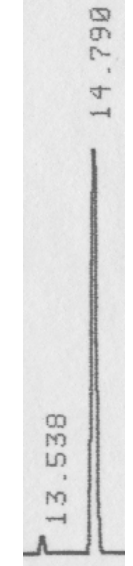

Product

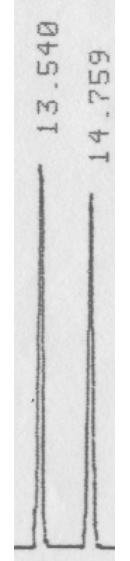

Racemate

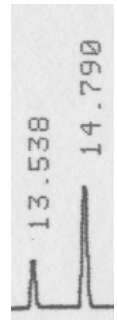

Product + Racemate

(3) Absolute configuration determined by comparison of derived Mosher's amide (vide infra) with authentic sample. 


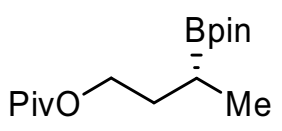

285.2. Found: 285.2

GC conditions: $120^{\circ} \mathrm{C}, 25 \mathrm{psi}, t_{S}=11.2 \mathrm{~min}, t_{R}=11.9 \mathrm{~min} ;$ oxidation/acylation product .

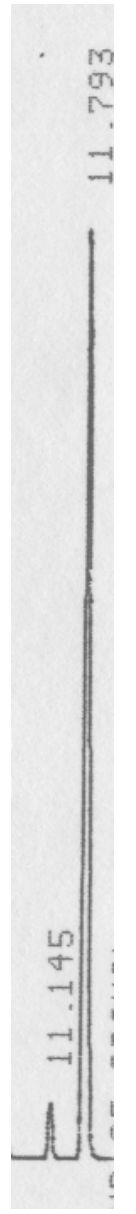

Product

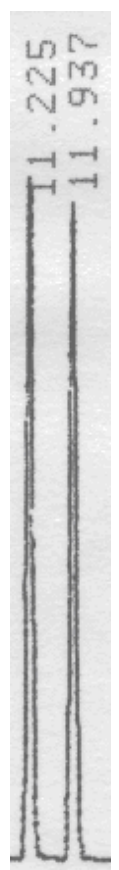

Racemate

(4) Absolute configuration determined by chiral GC analysis of protected commercially available (R)-(-)-1,3-butanediol. 


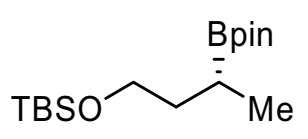

(R)-tert-butyldimethyl(3-(4,4,5,5-tetramethyl-1,3,2-dioxaborolan-2-yl)butoxy)silane $\mathbf{5}^{5}$ IR (neat): 3072 (s), 2997 (s), 2866 (m), 2306 (s), 1434 (s), $1293(\mathrm{~s}) \mathrm{cm}^{-1} .{ }^{1} \mathrm{H}$ NMR (400 $\left.\mathrm{MHz}, \mathrm{CDCl}_{3}\right): \delta 0.02(6 \mathrm{H}, \mathrm{s}), 0.86(9 \mathrm{H}, \mathrm{s}), 0.95(3 \mathrm{H}, \mathrm{d}, \mathrm{J}=7.6 \mathrm{~Hz}), 1.04-1.13(1 \mathrm{H}, \mathrm{m})$, $1.20(12 \mathrm{H}, \mathrm{s}), 1.42-1.52(1 \mathrm{H}, \mathrm{m}), 1.63-1.74(1 \mathrm{H}, \mathrm{m}), 3.53-3.65(2 \mathrm{H}, \mathrm{m}) .{ }^{13} \mathrm{C}$ NMR $\left(100.5 \mathrm{MHz}, \mathrm{CDCl}_{3}\right): \delta-4.8,15.9,18.8,23.0,25.1,25.2,26.4,36.5,63.0,83.2$. MS (ESI) $\mathrm{m} / \mathrm{z}$ calc'd for $\mathrm{C}_{16} \mathrm{H}_{36} \mathrm{BO}_{3} \mathrm{Si}: 315.2$. Found: 315.2 .

GC conditions: $110^{\circ} \mathrm{C}, 25 \mathrm{psi}, t_{S}=14.0 \mathrm{~min}, t_{R}=15.0 \mathrm{~min}$; oxidation/acylation product .

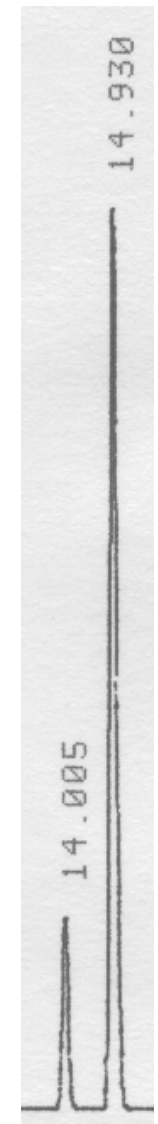

Product+Racemate

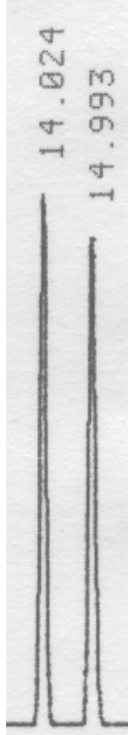

Racemate

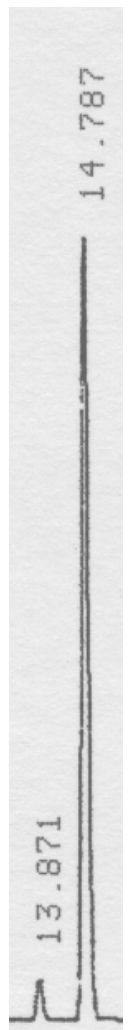

Product

(5) Absolute configuration determined by chiral GC analysis of protected commercially available (R)-(-)-1,3-butanediol. 
Page S- 8

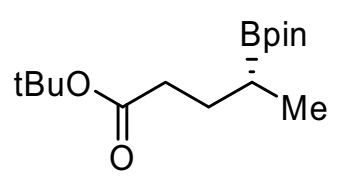

(R)-tert-butyl 4-(4,4,5,5-tetramethyl-1,3,2-dioxaborolan-2-yl)pentanoate. (neat): 2979 (s), 2933 (s), 2873 (s), 1735 (s), $1466(\mathrm{~m}), 1364(\mathrm{~m}), 1146(\mathrm{~m}) \mathrm{cm}^{-1} \cdot{ }^{1} \mathrm{H}$ NMR $\left(300 \mathrm{MHz}, \mathrm{CDCl}_{3}\right): \delta 0.94(3 \mathrm{H}, \mathrm{s}), 1.20(12 \mathrm{H}, \mathrm{s}), 1.40(9 \mathrm{H}, \mathrm{s}), 1.47-1.76(3 \mathrm{H}$, m), $2.20(2 \mathrm{H}, \mathrm{t}, \mathrm{J}=8.0 \mathrm{~Hz}) .{ }^{13} \mathrm{C} \mathrm{NMR}\left(100 \mathrm{MHz}, \mathrm{CDCl}_{3}\right): \delta 15.1,24.5,24.6,27.9$, 28.1, 34.7, 79.6, 82.7, 173.2. MS (ESI) $\mathrm{m} / \mathrm{z}$ calc'd for $\mathrm{C}_{15} \mathrm{H}_{29} \mathrm{BO}_{4} \mathrm{Na}^{+}$: 307.2. Found: 307.4 .

GC conditions: $120^{\circ} \mathrm{C}, 20 \mathrm{psi}, t_{S}=14.9 \mathrm{~min}, t_{R}=16.6 \mathrm{~min}$; oxidation/acylation product .

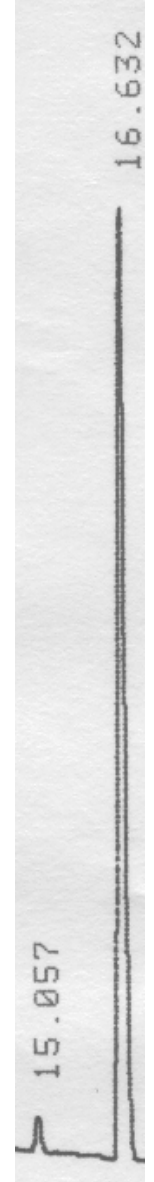

Product

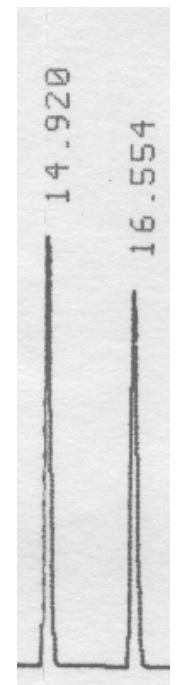

Racemate

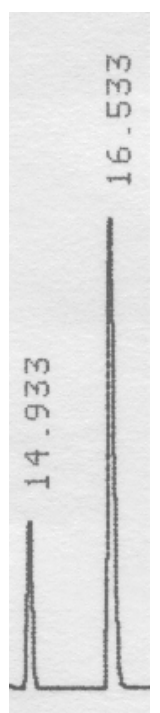

Product + Racemate 


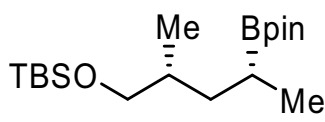

t-butyldimethyl((2R,4R)-2-methyl-4-(4,4,5,5-tetramethyl-1,3,2-dioxaborolan-2yl)pentyloxy)silane ${ }^{6}$ : IR (neat): 2977 (s), 2929 (s), 2856 (s), 1472 (m), 1387 (s), 1315 (s), 1256 (s), 1146 (s), $1094(\mathrm{~s}), 837 \mathrm{~cm}^{-1}$. ${ }^{1} \mathrm{H}$ NMR (400 MHz, $\left.\mathrm{CDCl}_{3}\right): \delta 0.02(6 \mathrm{H}$, s), 0.82-1.15 (17H, m), $1.23(12 \mathrm{H}, \mathrm{s}), 1.45-1.67(2 \mathrm{H}, \mathrm{m}), 3.25(1 \mathrm{H}, \mathrm{dd}, \mathrm{J}=9.9,7.2 \mathrm{~Hz})$, $3.45(1 \mathrm{H}, \mathrm{dd}, \mathrm{J}=9.6,5.1 \mathrm{~Hz}) .{ }^{13} \mathrm{C}$ NMR $\left(100.5 \mathrm{MHz}, \mathrm{CDCl}_{3}\right): \delta-5.5(2 \mathrm{C}), 15.1,16.4$, 18.2, 24.5 (2C), 25.8, 34.2, 36.0, 68.5, 82.6. MS (ESI) m/z calc'd for $\mathrm{C}_{18} \mathrm{H}_{39} \mathrm{BO}_{3} \mathrm{SiNa}^{+}:$365.3. Found: 365.4 .

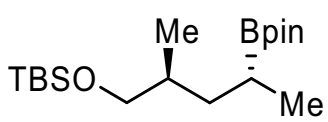

t-butyldimethyl((2S,4R)-2-methyl-4-(4,4,5,5-tetramethyl-1,3,2-dioxaborolan-2yl)pentyloxy)silane ${ }^{6}$ : IR (neat): 2979 (s), 2931 (s), 2858 (s), 1459 (m), 1372 (s), 1318 (s), 1256 (s), 1146 (s), 1094 (s), 837 (s) $\mathrm{cm}^{-1} .{ }^{1} \mathrm{H}$ NMR (300 MHz, $\left.\mathrm{CDCl}_{3}\right): \delta .0 .02$ $(6 \mathrm{H}, \mathrm{s}), 0.82-1.15(17 \mathrm{H}, \mathrm{m}), 1.23(12 \mathrm{H}, \mathrm{s}), 1.45-1.67(2 \mathrm{H}, \mathrm{m}), 3.25(1 \mathrm{H}, \mathrm{dd}, \mathrm{J}=9.9$, $7.2 \mathrm{~Hz}), 3.45(1 \mathrm{H}, \mathrm{dd}, \mathrm{J}=9.6,5.1 \mathrm{~Hz}) .{ }^{13} \mathrm{C} \mathrm{NMR}\left(100.5 \mathrm{MHz}, \mathrm{CDCl}_{3}\right): \delta-5.5(2 \mathrm{C})$, 16.0, 16.9, 18.2, 24.5 (2C), 25.8, 34.7, 36.8, 68.4, 82.6. MS (ESI) m/z calc'd for $\mathrm{C}_{18} \mathrm{H}_{39} \mathrm{BO}_{3} \mathrm{SiNa}^{+}: 365.3$. Found: 365.4 ..

(6) Relative stereochemistry verified by comparison of corresponding diol with literature ${ }^{1} \mathrm{H}$ NMR data: Chiarello, J.; Joullie, M. M. Synth. Commun. 1989, 19, 3379. 
<smiles>C=C(Br)C1CCCCC1</smiles>

1
1. $5 \mathrm{~mol} \% \mathrm{Rh}(\mathrm{nbd})_{2} \mathrm{BF}_{4}$ $8 \mathrm{~mol} \%$ Walphos 1 $\mathrm{H}_{2}, 35 \mathrm{~atm}$ $-35^{\circ} \mathrm{C}$ 2. $\mathrm{BCl}_{3}$, then $\mathrm{BnN}_{3}$ $22{ }^{\circ} \mathrm{C}$<smiles>CC(Cc1ccccc1)C1CCCCC1</smiles>

2

Synthesis of (R)-N-benzyl-1-cyclohexylethanamine: A vial was charged with bis(norbornadiene)rhodium(I) tetrafluoroborate $(9.5 \mathrm{mg}, 0.025 \mathrm{mmol}, 0.05$ equiv), Walphos 1 (38 mg, $0.041 \mathrm{mmol}, 0.08$ equiv) and 1,2dichloroethane $(2.4 \mathrm{ml})$ in a dry-box and stirred at room temperature for 1 minute. 2-(1-Cyclohexyl-vinyl)4,4,5,5-tetramethyl-[1,3,2] dioxaborolane $(120 \mathrm{mg}, 0.51 \mathrm{mmol}, 1$ equiv) was added to the catalyst solution and the vial was capped and removed from the dry-box. The cap was removed and the vial placed in a Parr Instruments pressure vessel. The vessel was partly submerged in a cryo-bath and cooled to approx. $-35^{\circ} \mathrm{C}$, then purged with $\mathrm{H}_{2}$. The vessel was charged with 30 bar $\mathrm{H}_{2}$ and then depressurized. The vessel was re-charged with $30 \mathrm{bar} \mathrm{H}_{2}$ and left stirring for $15 \mathrm{~h}$. The pressure was released and the vessel removed from the cryo-bath. The vial was taken from the pressure vessel, capped, and allowed to warm to ambient temperature. The vial was then taken back into the dry-box and boron trichloride $\left(1 \mathrm{M}\right.$ in $\mathrm{CH}_{2} \mathrm{Cl}_{2}, 1.0 \mathrm{ml}, 1.0 \mathrm{mmol}, 2$ equiv) was added. After $3 \mathrm{~h}$, benzyl azide $(0.16 \mathrm{ml}, 1.3 \mathrm{mmol}, 2.5$ equiv) was added carefully (nitrogen evolution observed) and the mixture stirred for a further $4 \mathrm{~h}$. The vial was removed from the dry-box and aqueous $\mathrm{NaOH}$ solution $(2 \mathrm{M}$, $1 \mathrm{ml}$ ) was added. The mixture was stirred for $0.5 \mathrm{~h}$ at ambient temperature, and then extracted with EtOAc. Evaporation of the volatiles in vacuo provided the crude residue, which was purified by flash chromatography (9:1 hexanes/EtOAc) to provide a colorless oil $(91 \mathrm{mg}, 82 \%)$. Spectral data are in agreement with that reported for this compound. ${ }^{7} \mathrm{H}$ NMR (400 MHz, CDCl3): $\delta 0.95(3 \mathrm{H}, \mathrm{d}, \mathrm{J}=5.3 \mathrm{~Hz}), 1.00-1.32(7 \mathrm{H}, \mathrm{m}), 1.50-1.72(5 \mathrm{H}$, $\mathrm{m}), 2.40-2.44(1 \mathrm{H}, \mathrm{m}), 3.62(1 \mathrm{H}, \mathrm{d}, \mathrm{J}=13.2 \mathrm{~Hz}), 3.76(1 \mathrm{H}, \mathrm{d}, \mathrm{J}=13.2 \mathrm{~Hz}), 7.18-7.27(5 \mathrm{H}, \mathrm{m}) .{ }^{13} \mathrm{C} \mathrm{NMR}$ $\left(100.5 \mathrm{MHz}, \mathrm{CDCl}_{3}\right): \delta 17.1,27.0,26.8,26.9,28.3,30.1,43.2,51.8,57.2,127.0,128.4,128.6,141.3$.

${ }^{1}$ H NMR of debenzylated Mosher's amide; NH resonance:

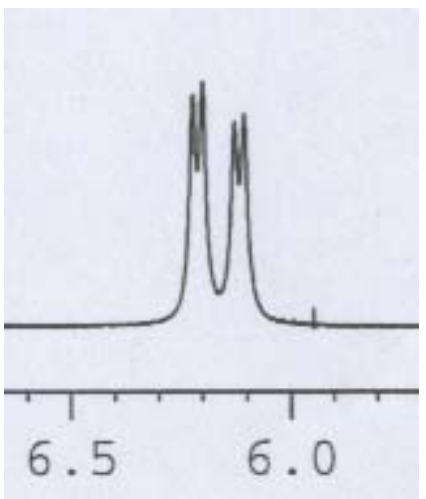

racemic

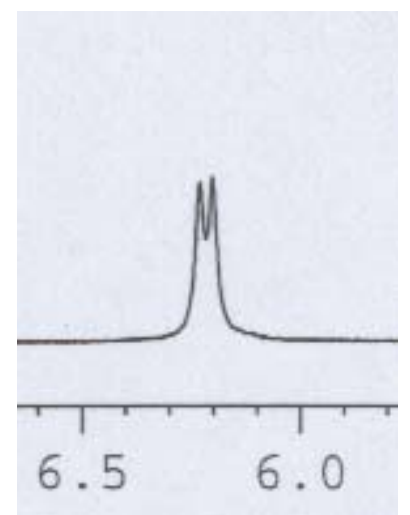

reaction product

(7) Data consistent with literature: Majewski, M.; Gleave, D. M. J. Org. Chem. 1992, 57, 3599. 


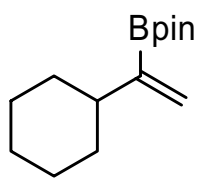

1
1. $5 \mathrm{~mol} \% \mathrm{Rh}(\mathrm{nbd})_{2} \mathrm{BF}_{4}$ $8 \mathrm{~mol} \%$ Walphos 1 $\mathrm{H}_{2}, 35 \mathrm{~atm}$ $-35^{\circ} \mathrm{C}$

2. $\mathrm{ClCH}_{2} \mathrm{Li}$, THF $-78^{\circ} \mathrm{C}$

3. $\mathrm{NaOH}, \mathrm{H}_{2} \mathrm{O}_{2}$

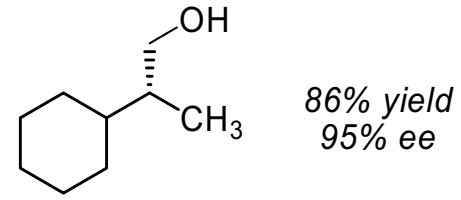

3

Synthesis of (R)-2-cyclohexylpropan-1-ol: A vial was charged with bis(norbornadiene)rhodium(I) tetrafluoroborate $(9.5 \mathrm{mg}, 0.025 \mathrm{mmol}, 0.05$ equiv), Walphos 1 (38 $\mathrm{mg}, 0.041 \mathrm{mmol}, 0.08$ equiv) and 1,2dichloroethane $(2.4 \mathrm{ml})$ in a dry-box and stirred at room temperature for 1 minute. 2-(1-Cyclohexyl-vinyl)4,4,5,5-tetramethyl-[1,3,2] dioxaborolane $(120 \mathrm{mg}, 0.51 \mathrm{mmol}, 1$ equiv) was added to the catalyst solution and the vial was capped and removed from the dry-box. The cap was removed and the vial placed in a Parr Instruments pressure vessel. The vessel was partly submerged in a cryo-bath and cooled to approx. $-35^{\circ} \mathrm{C}$, then purged with $\mathrm{H}_{2}$. The vessel was charged with 30 bar $\mathrm{H}_{2}$ and then depressurized. The vessel was re-charged with 30 bar $\mathrm{H}_{2}$ and left stirring for $15 \mathrm{~h}$. The pressure was released and the vessel removed from the cryo-bath. The vial was taken from the pressure vessel and the solvent removed in vacuo. THF ( $2 \mathrm{ml})$ was added followed by bromochloromethane ( $43 \mu \mathrm{l}, 0.66 \mathrm{mmol}, 1.3$ equiv). The reaction mixture was then cooled to $-78{ }^{\circ} \mathrm{C}$ and $\mathrm{n}$ $\operatorname{BuLi}(2.5 \mathrm{M}$ in hexanes, $0.26 \mathrm{ml}, 0.66 \mathrm{mmol}, 1.3$ equiv) added dropwise. The reaction was allowed to warm to ambient temperature overnight and then $30 \%$ aqueous hydrogen peroxide solution $(0.5 \mathrm{ml})$ and aqueous $\mathrm{NaOH}$ solution $(2 \mathrm{M}, 0.5 \mathrm{ml})$ were added. After a further $2.5 \mathrm{~h}$, the reaction mixture was extracted with EtOAc and concentrated in vacuo. Purification by flash chromatography (9:1 hexanes/EtOAc) provided the product as a colorless oil $(62 \mathrm{mg}, 85 \%)$. Spectral data are in agreement with that reported for this compound. ${ }^{8}{ }^{1} \mathrm{H}$ NMR $\left(300 \mathrm{MHz}, \mathrm{CDCl}_{3}\right): \delta 0.88(3 \mathrm{H}, \mathrm{d}, \mathrm{J}=6.9 \mathrm{~Hz}), 0.90-1.40(6 \mathrm{H}, \mathrm{m}), 1.40-1.55(1 \mathrm{H}, \mathrm{m}), 1.60-1.80(5 \mathrm{H}, \mathrm{m}), 3.43$ $(1 \mathrm{H}, \mathrm{dd}, \mathrm{J}=10.5,6.9 \mathrm{~Hz}), 3.59(1 \mathrm{H}, \mathrm{dd}, \mathrm{J}=10.5,5.7 \mathrm{~Hz}) .{ }^{13} \mathrm{C} \mathrm{NMR}\left(75 \mathrm{MHz}, \mathrm{CDCl}_{3}\right): \delta 13.4,26.8,26.9$, $27.0,29.0,31.2,39.6,41.2,66.5$.

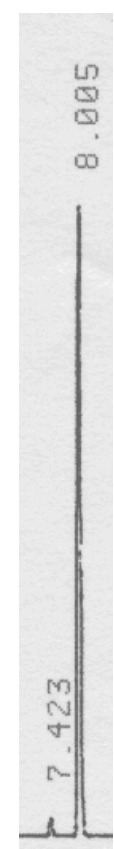

Product

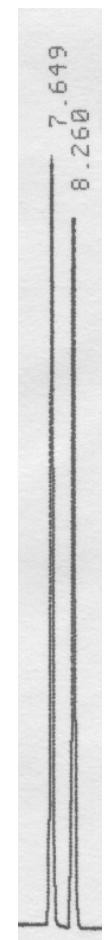

Racemate

(8) Data consistent with literature: Kondakov, D. Y.; Negishi, E.-I. J. Am. Chem. Soc. 1995, 117, 10771. 
Page S-12

$600^{\circ} D T$

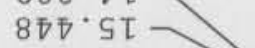

$985^{\circ} \mathrm{2Z}$

$5 \angle 9^{\circ} \nabla Z$

$\nabla 0 L$ DZ

$\angle S L \cdot \nabla Z$

$288 \cdot 82$

$787^{\circ} 62$

$878^{\circ}$ Tह

$L T \zeta \cdot \varepsilon \varepsilon$

$6 \varepsilon 9 \cdot 9 L$

$\angle S 6 \cdot 9 L$

$Z 62^{\circ} \cdot L L$

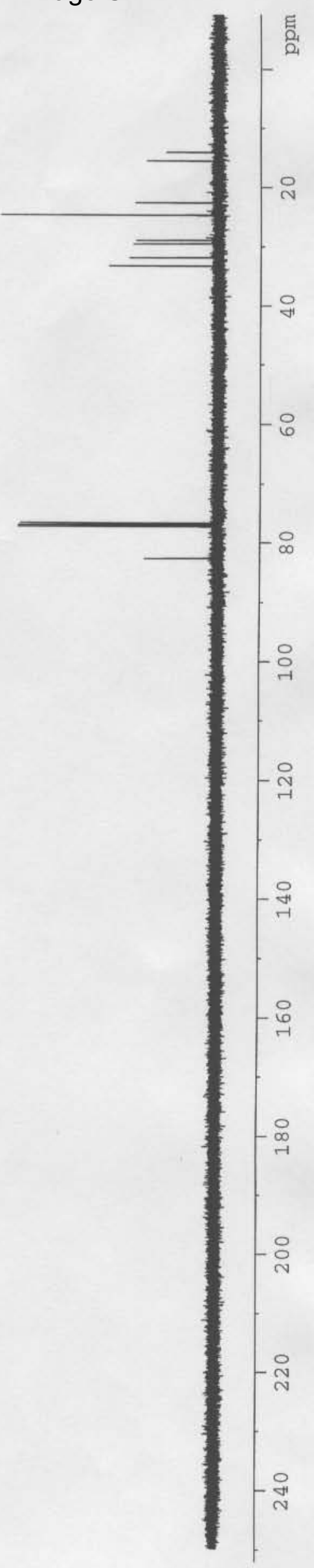

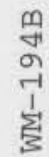

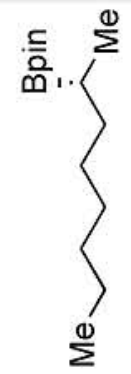


Page S-13

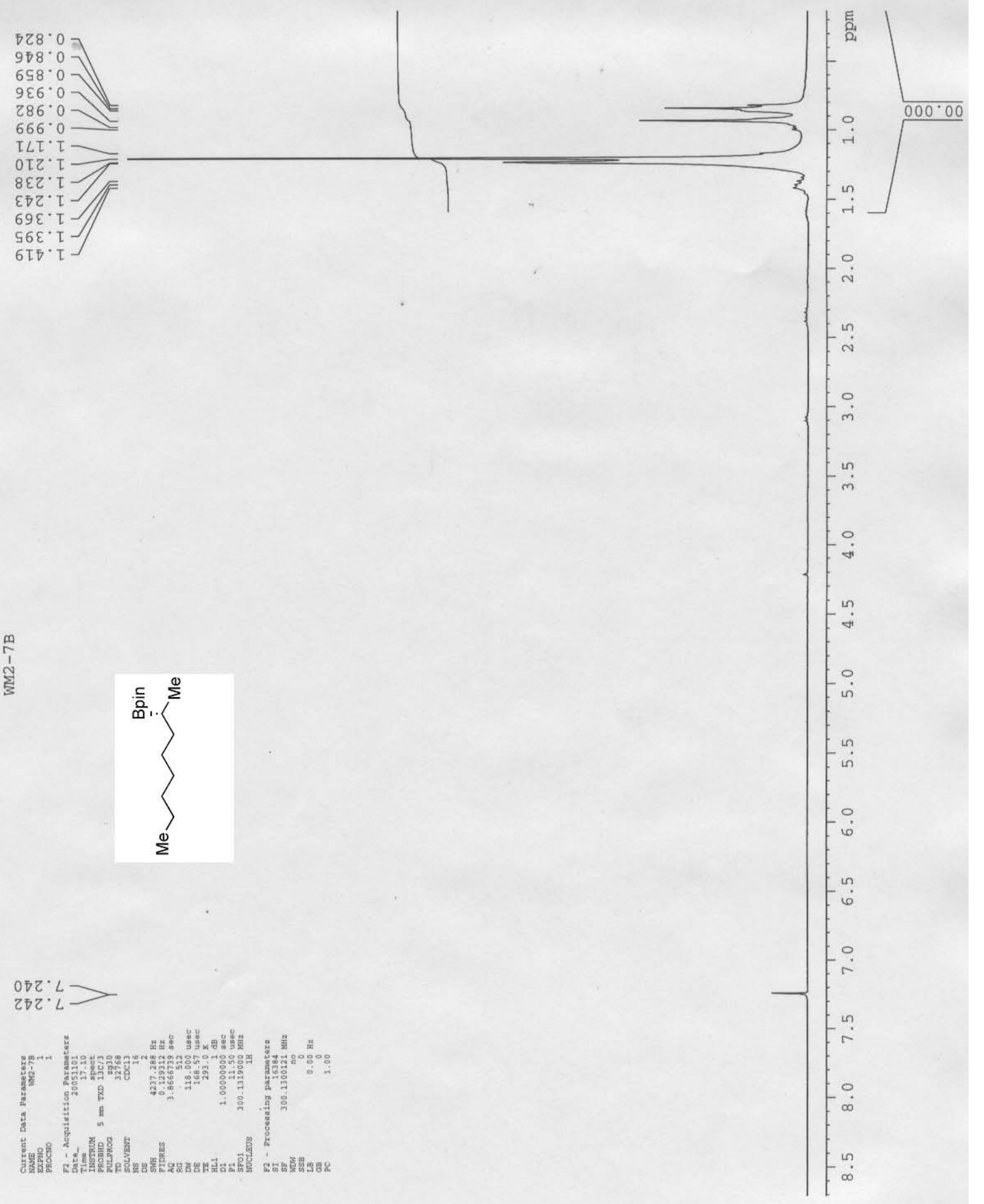


Page S-14

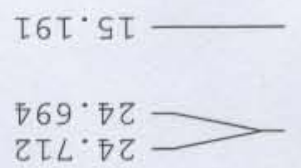

$8 L 6^{\circ} 8 \varepsilon$
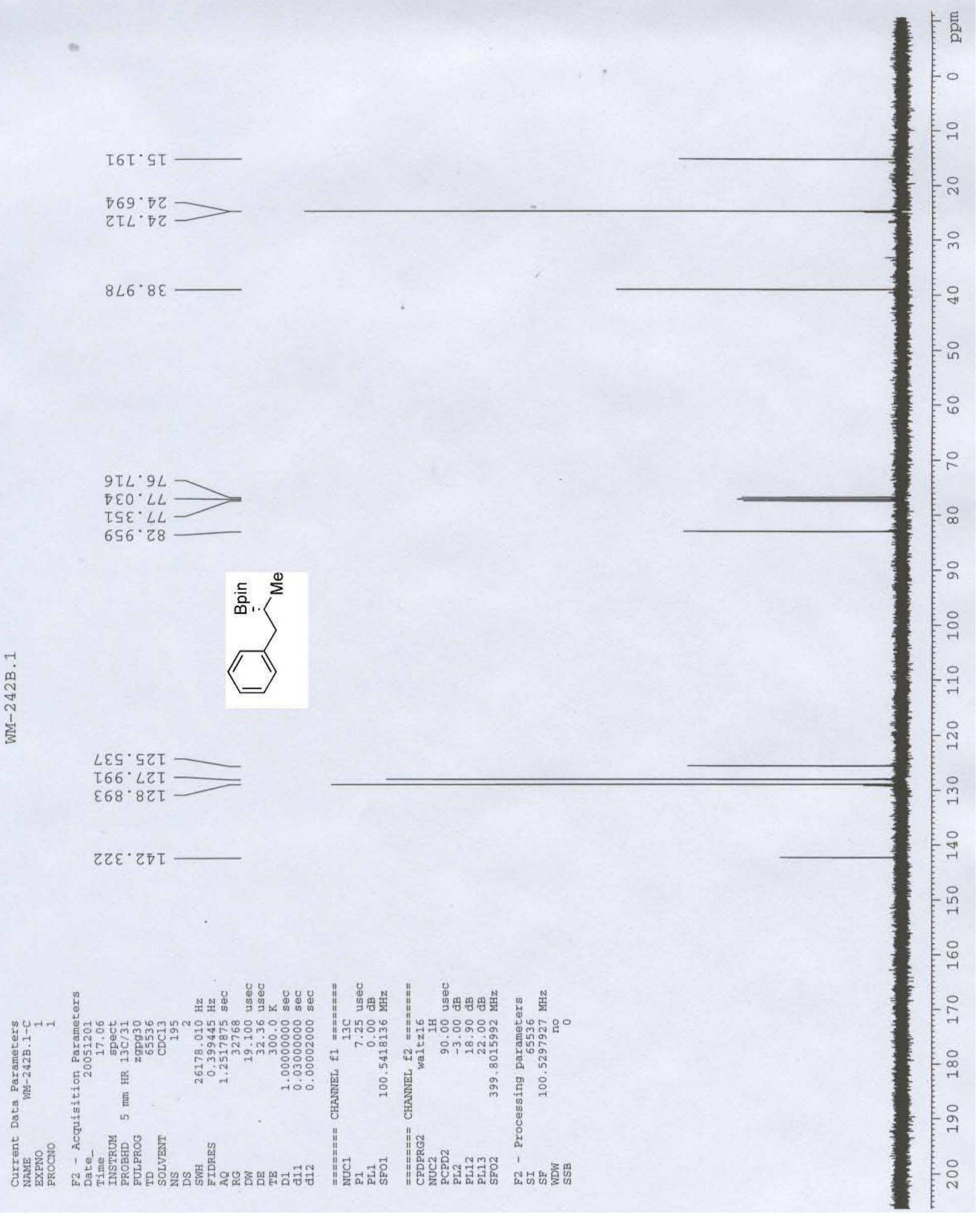
Page S-15

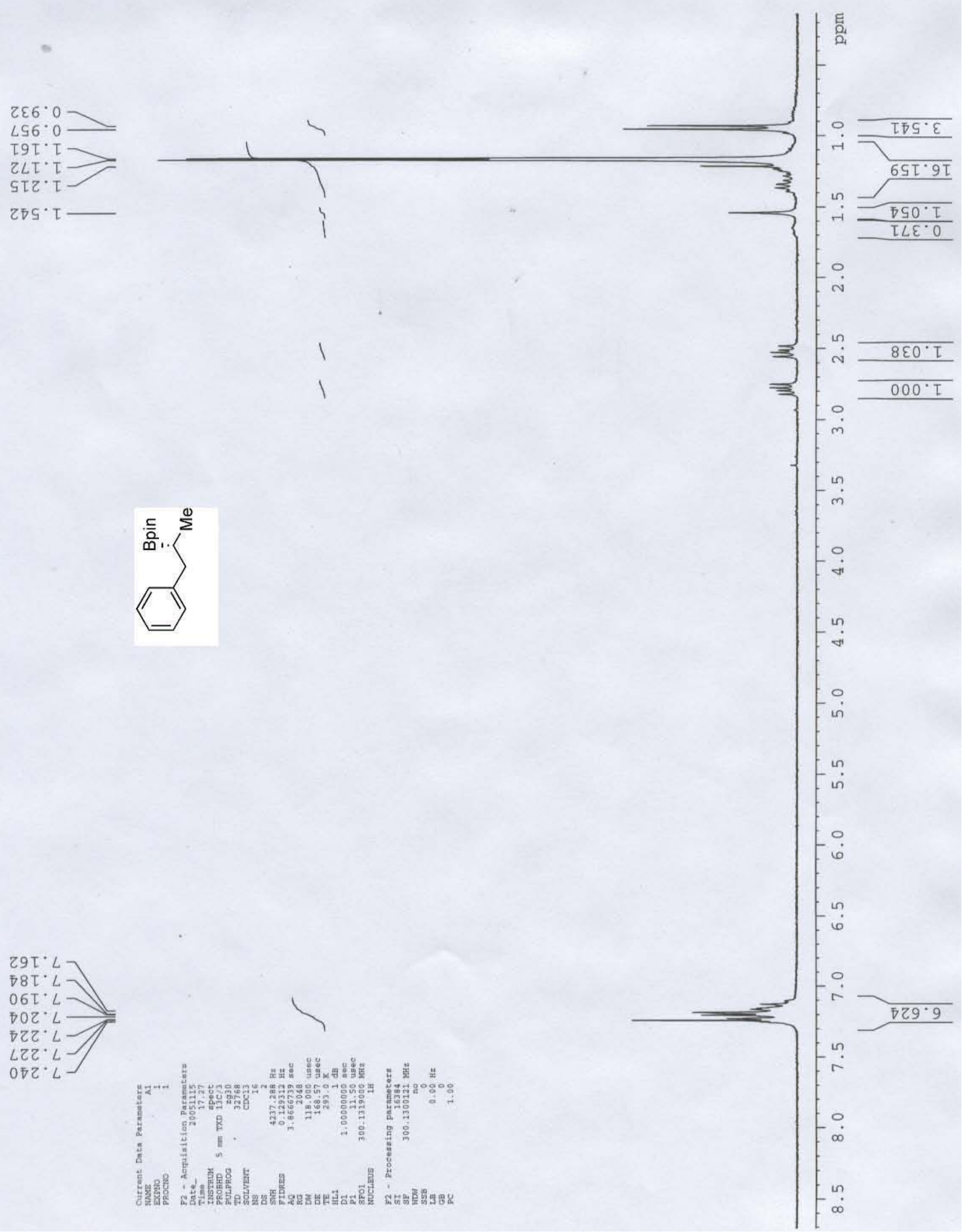




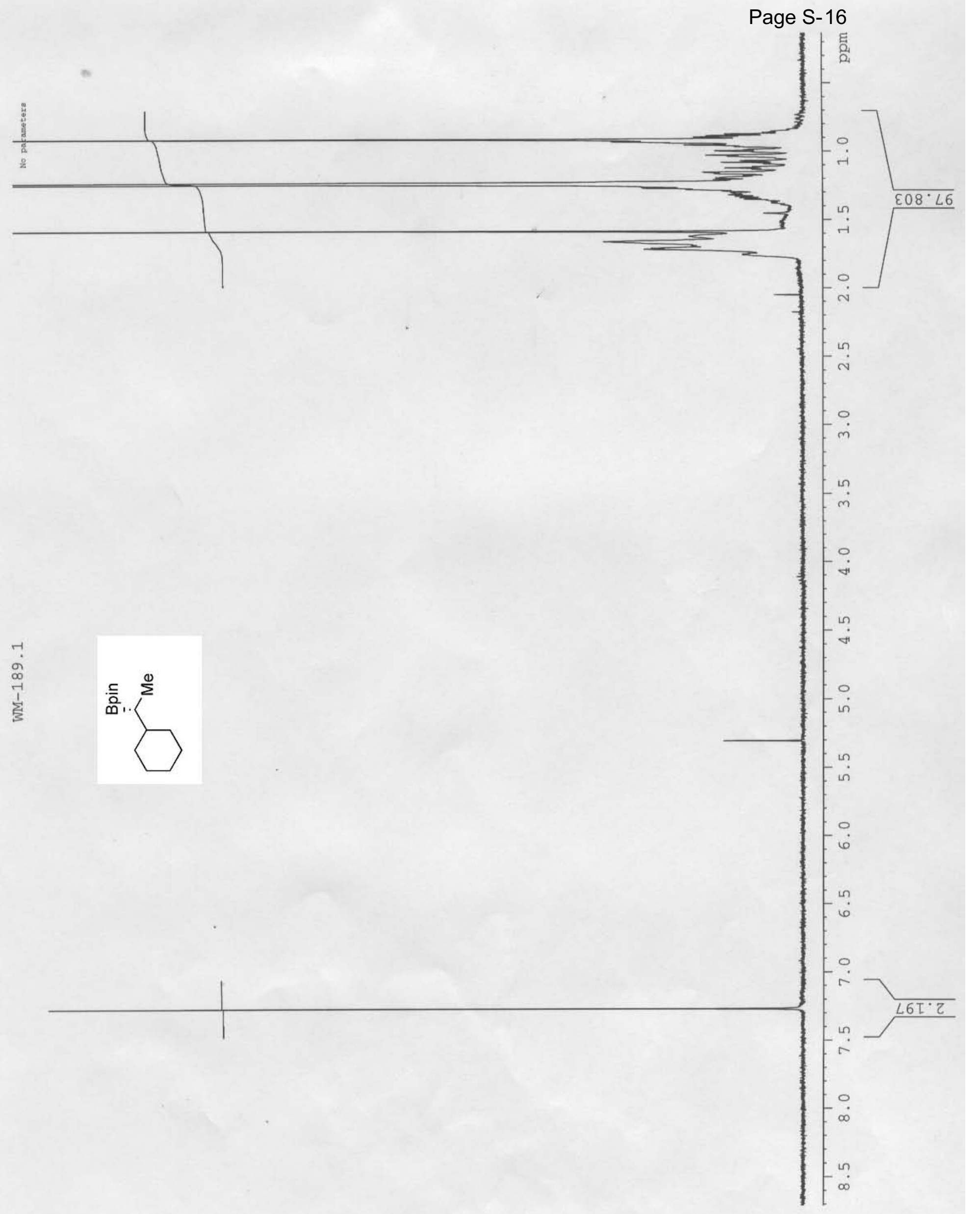




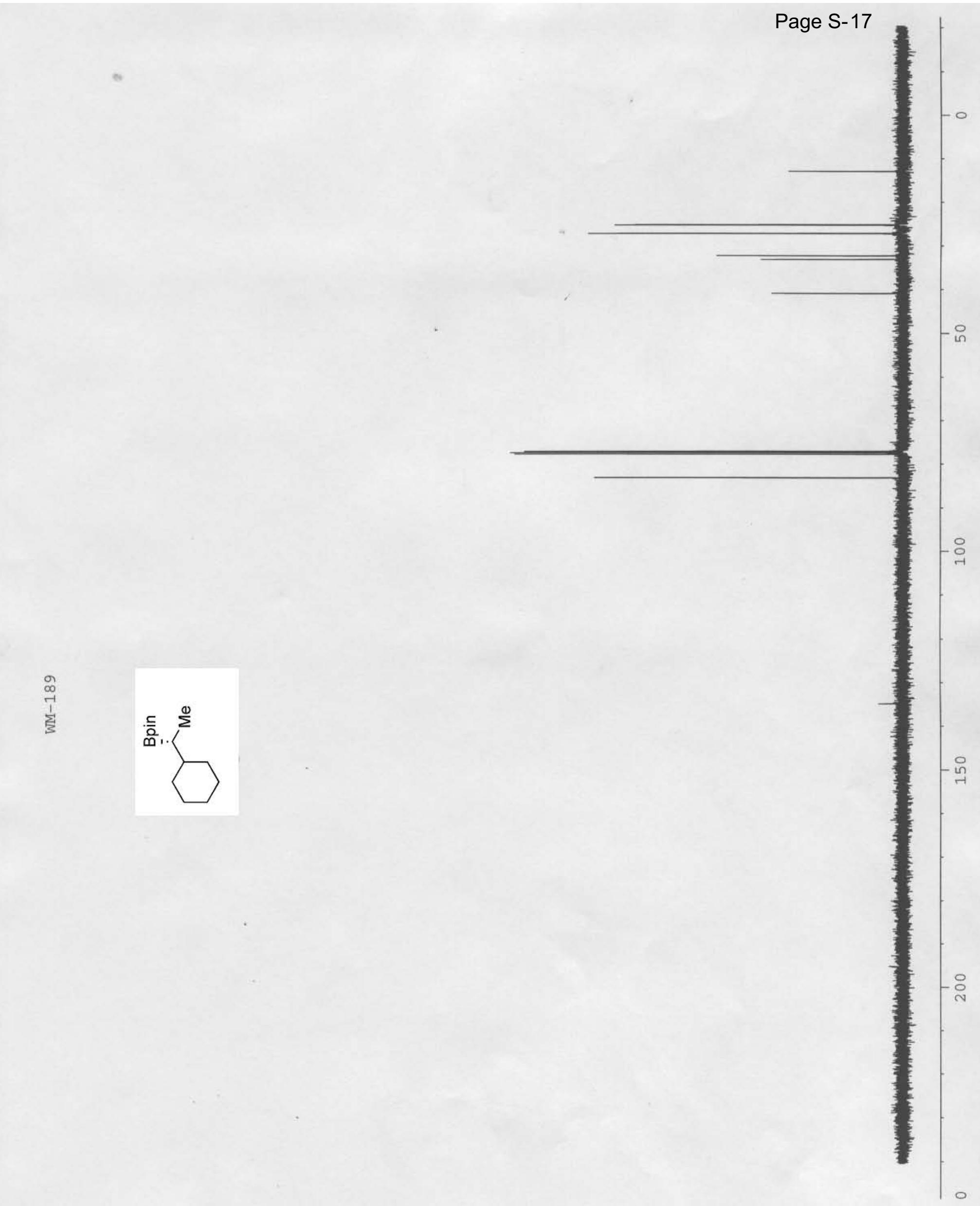


Page S-18

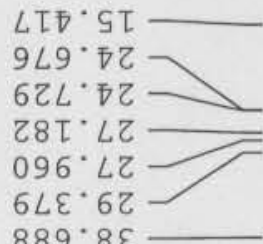

$729 \cdot 79$

$8 \angle 9^{\circ} 9 L$ $566^{\circ} 9 L$

โโ $\varepsilon^{\circ} L L$

$\angle 98^{\circ} 28^{\circ}$

4
ㄱ.
1
तै
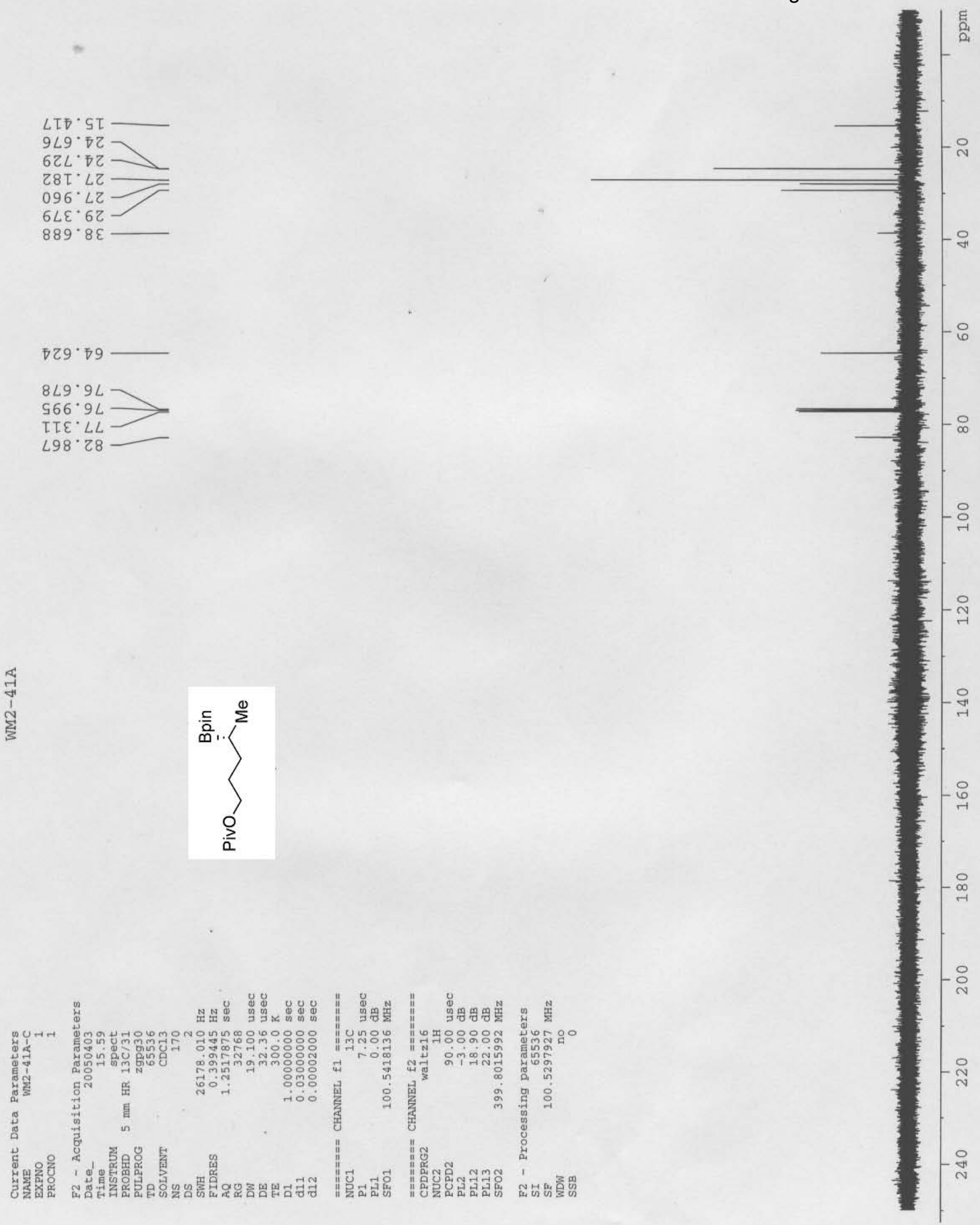
Page S-19

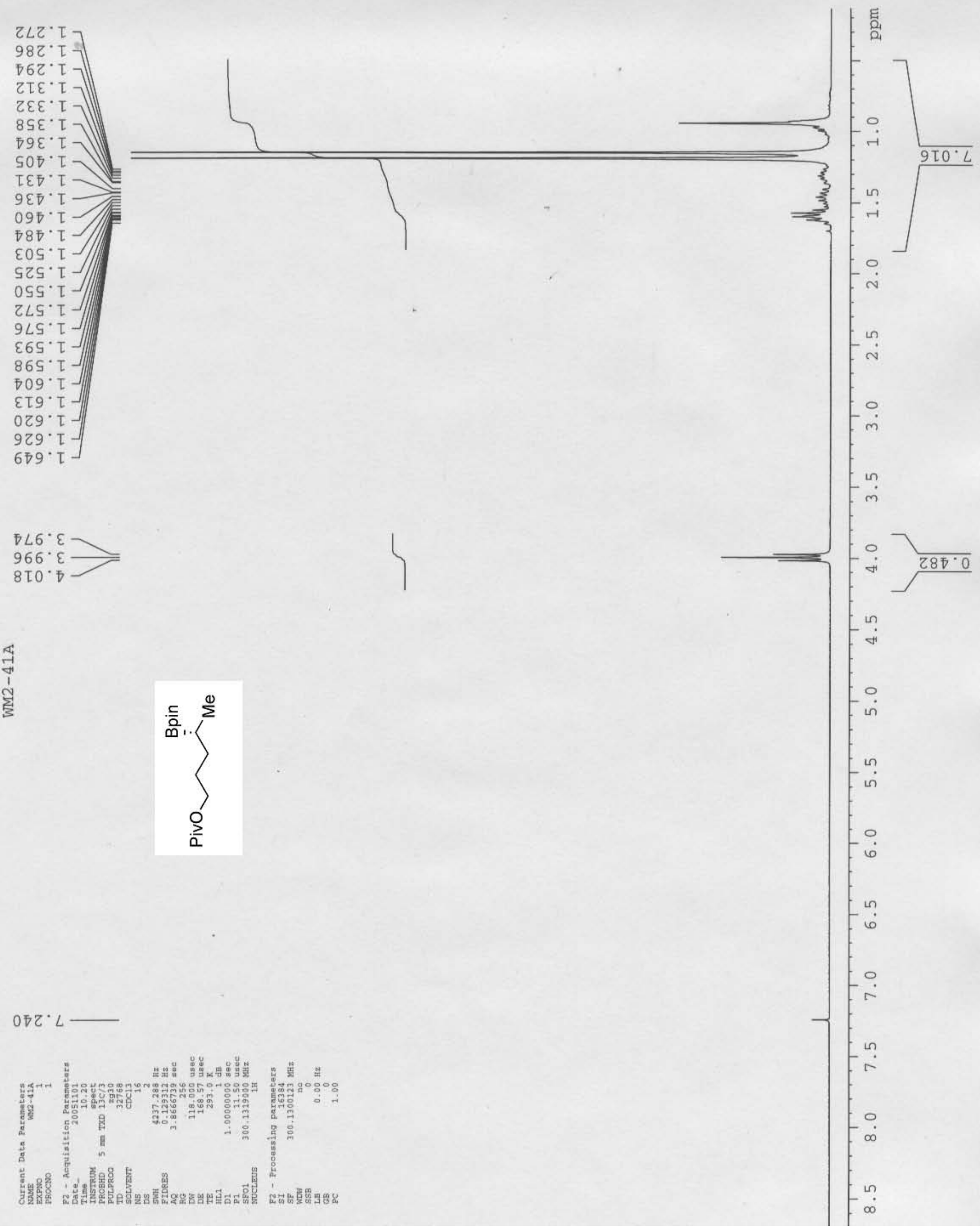


Page S-20
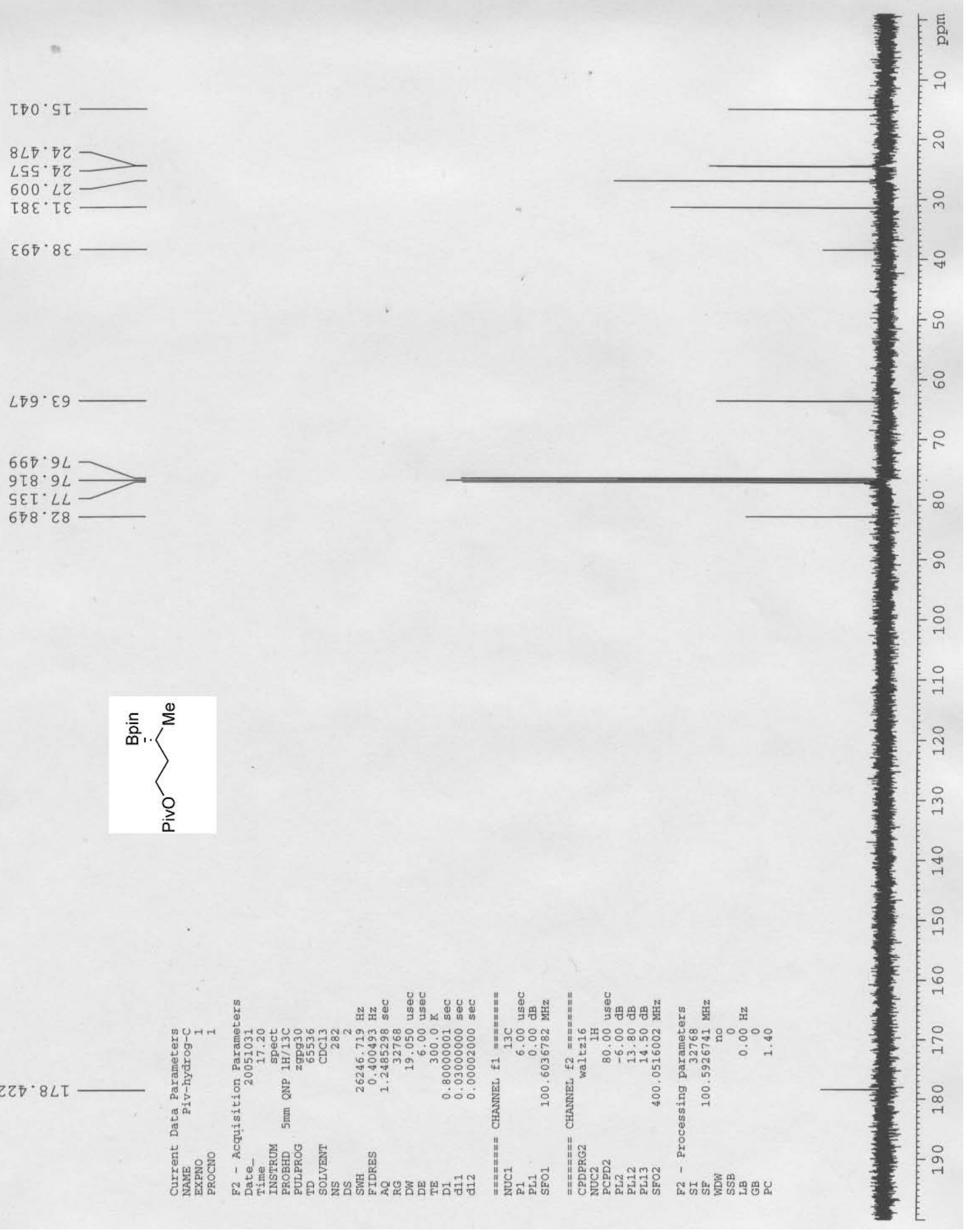
Page S-21

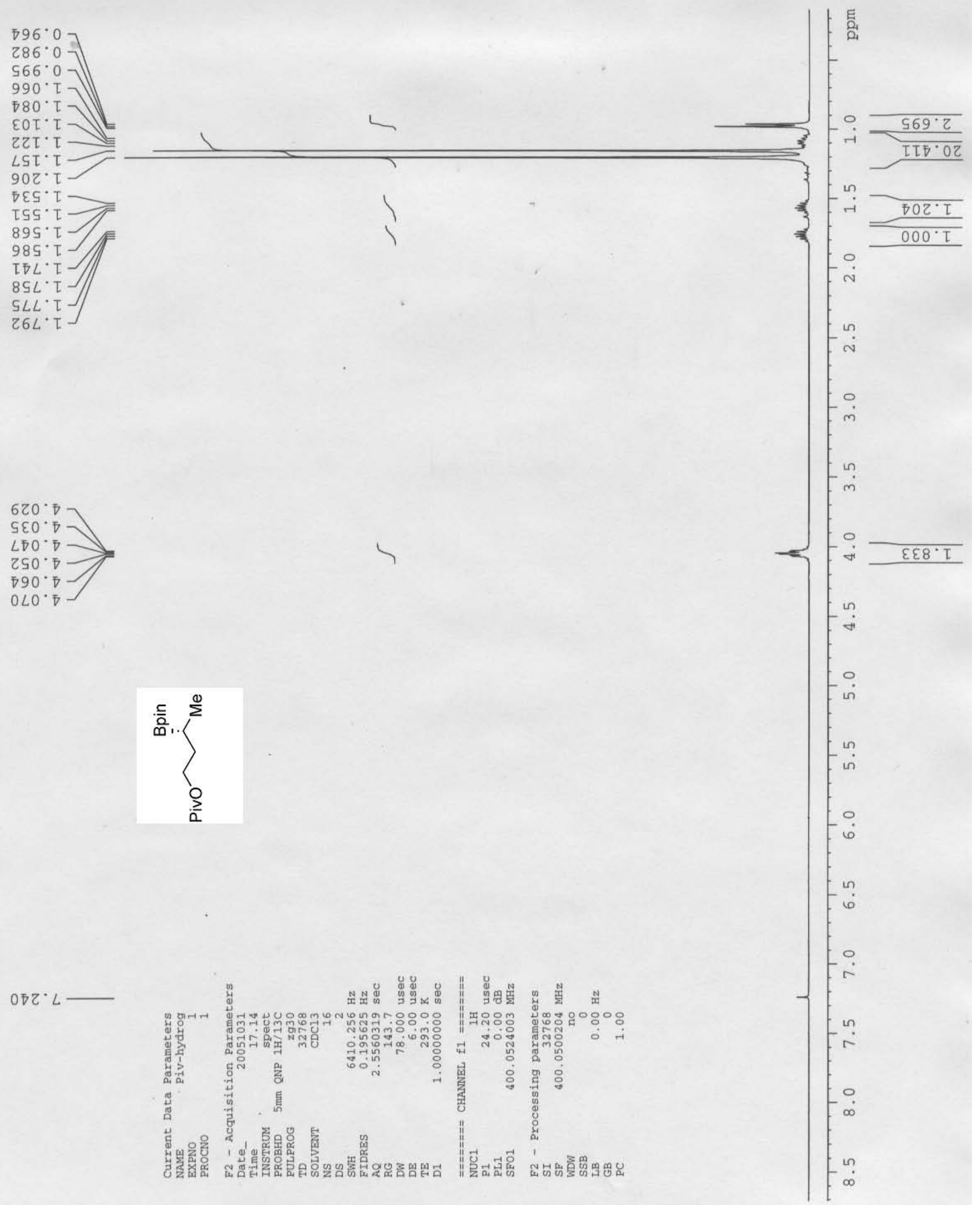




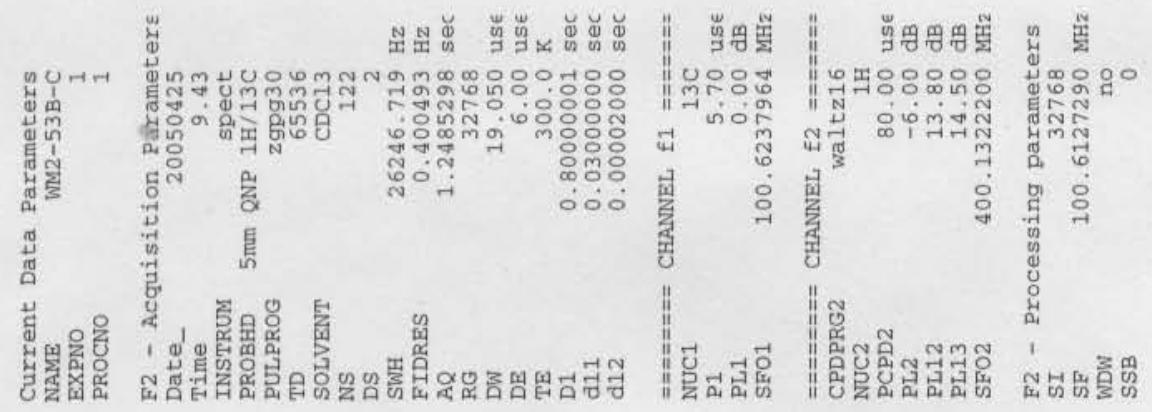

Page S-22

¿ร8. ५ โ

$06 L \cdot 8 T$

$670^{\circ} \varepsilon$

ع $60^{\circ} \mathrm{\varsigma} \mathrm{Z}$

$69 T^{\circ} \mathrm{sZ}$

$607 \cdot 92$

OZS. $9 \varepsilon$

m

$\varsigma \nabla 0 \cdot \varepsilon 9$

$680^{\circ} \angle L$

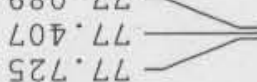

$9 z Z \cdot \varepsilon 8$

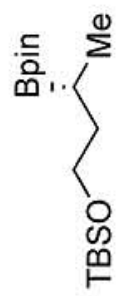


Page S-23

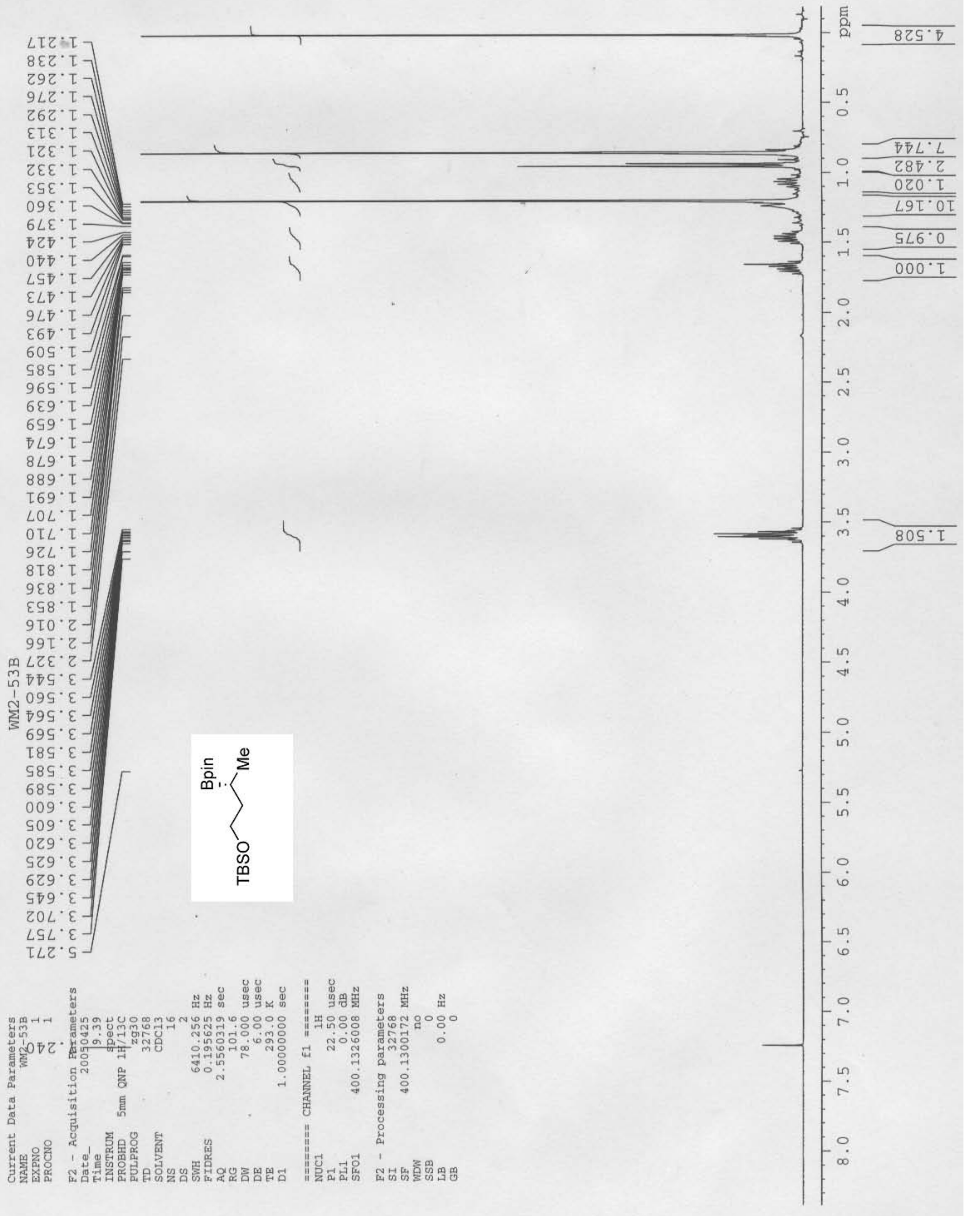


Page S-24

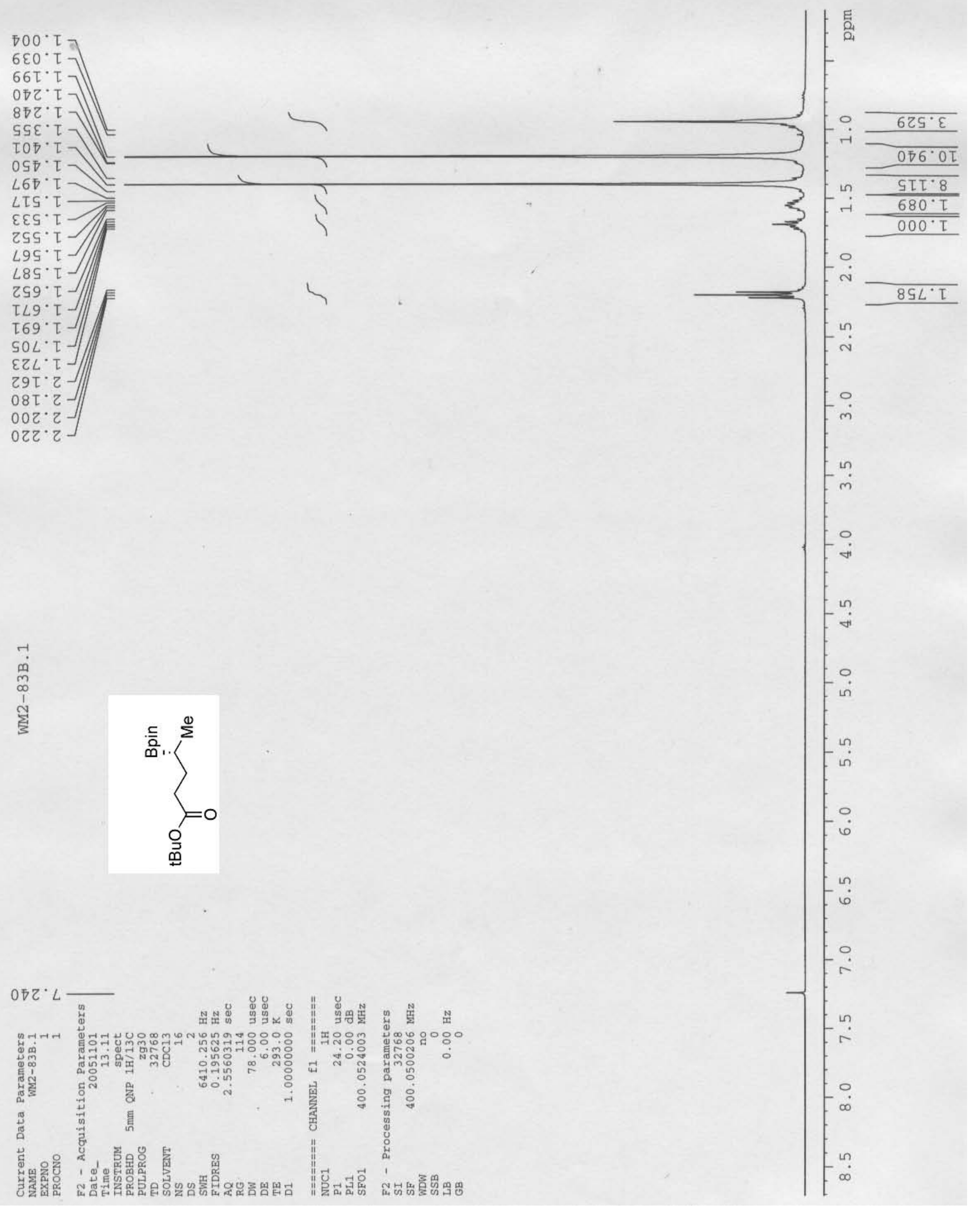


Page S-25

OSO SI
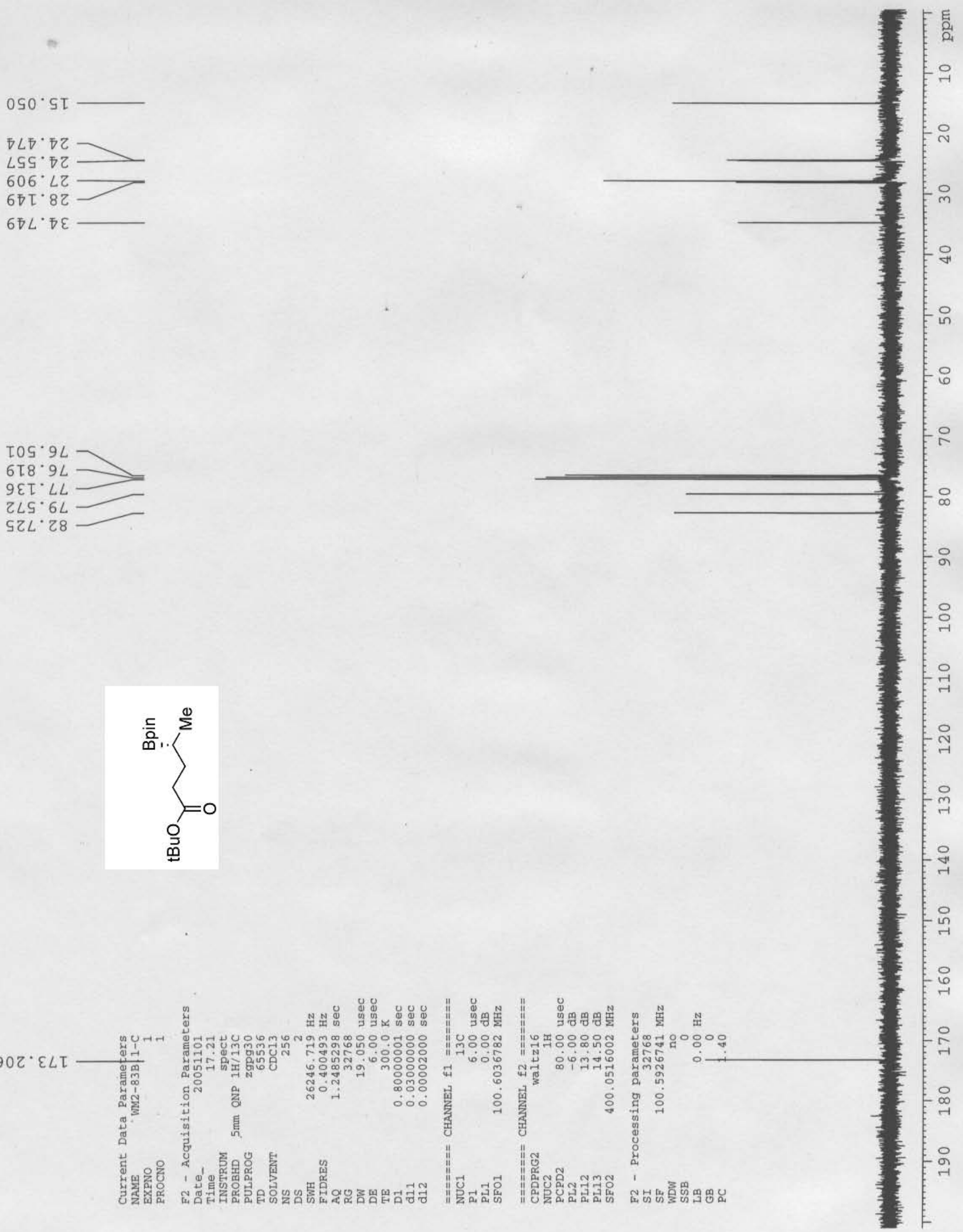

IOS. $9 L$

6 I $.9 L$

$9 \varepsilon \tau^{\circ} L L$

ZLS. $6 L$

$\Longrightarrow$

$\mathrm{SZL} \cdot \mathrm{Z} 8$

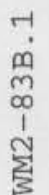<smiles>C[13CH][13C](=O)CCC(=O)O</smiles> 
Page S-26

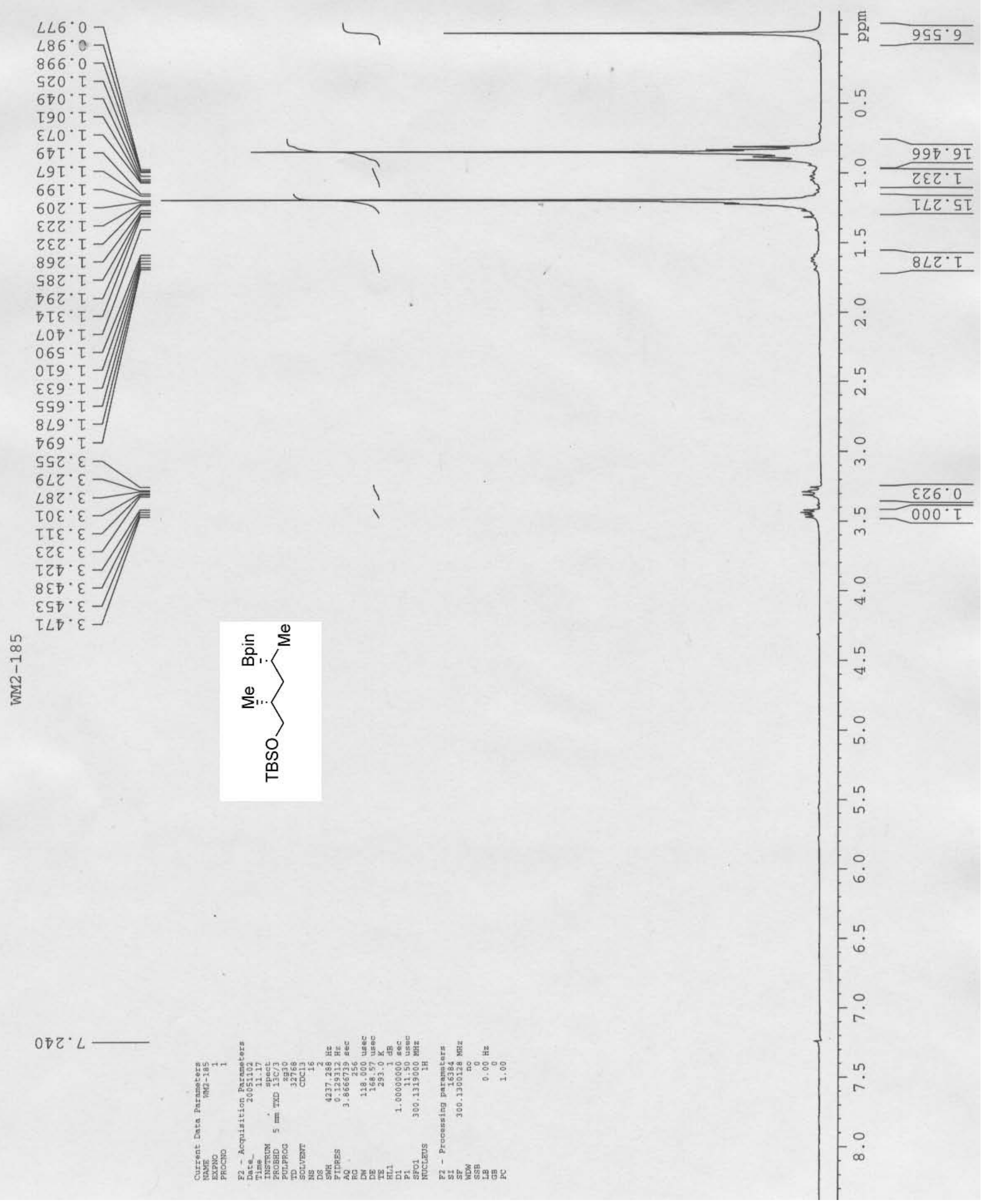


Page S-27
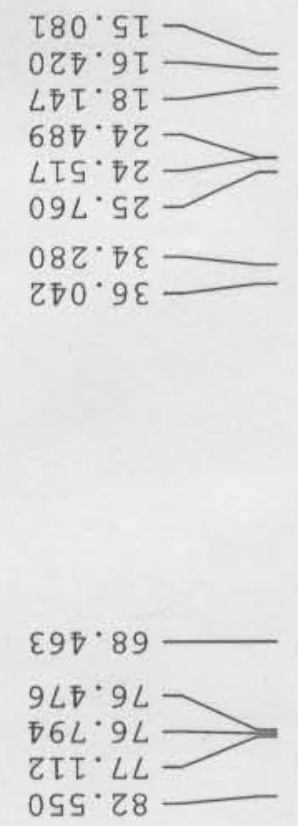

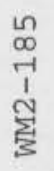
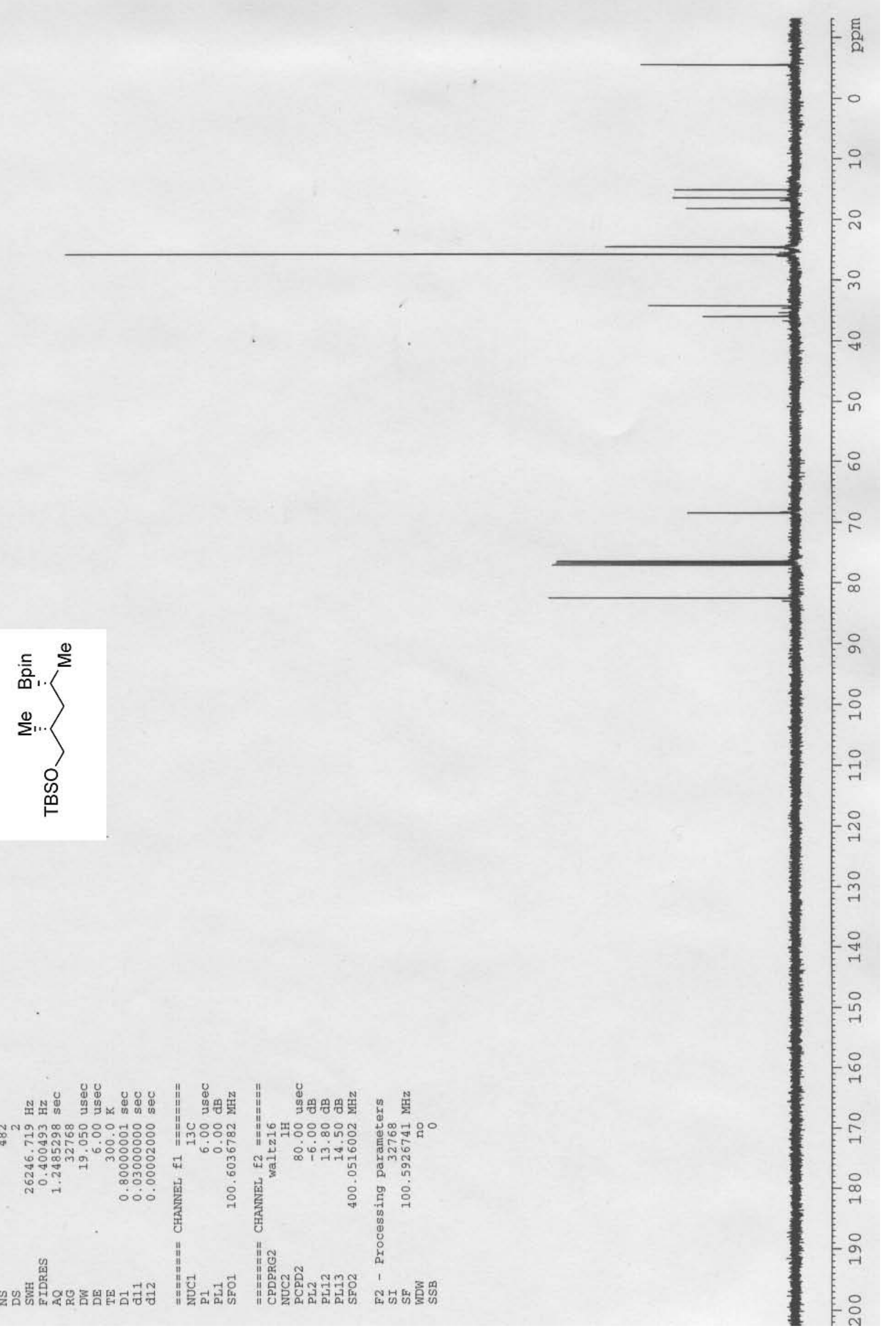
Page S-28

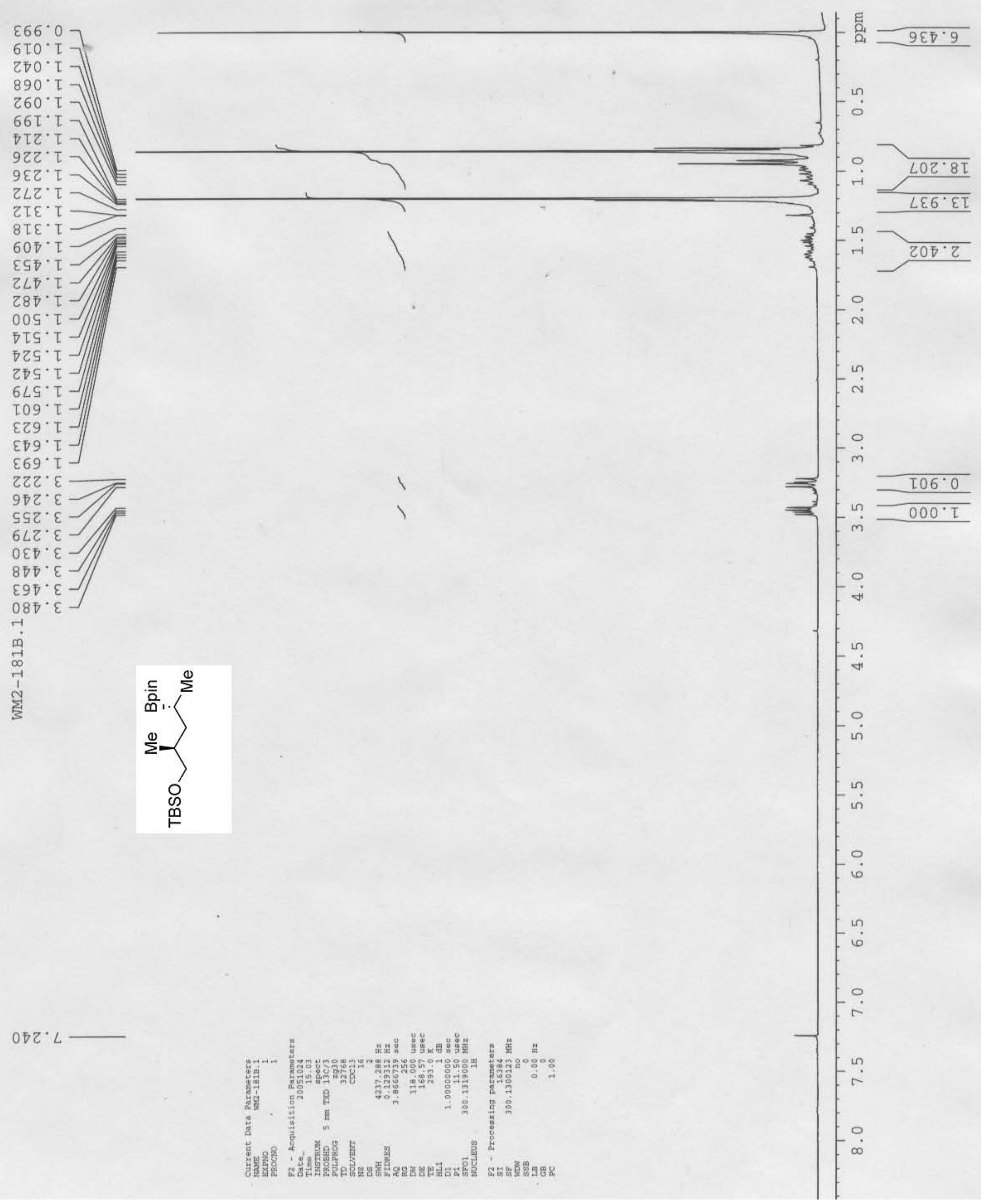


Page S-29
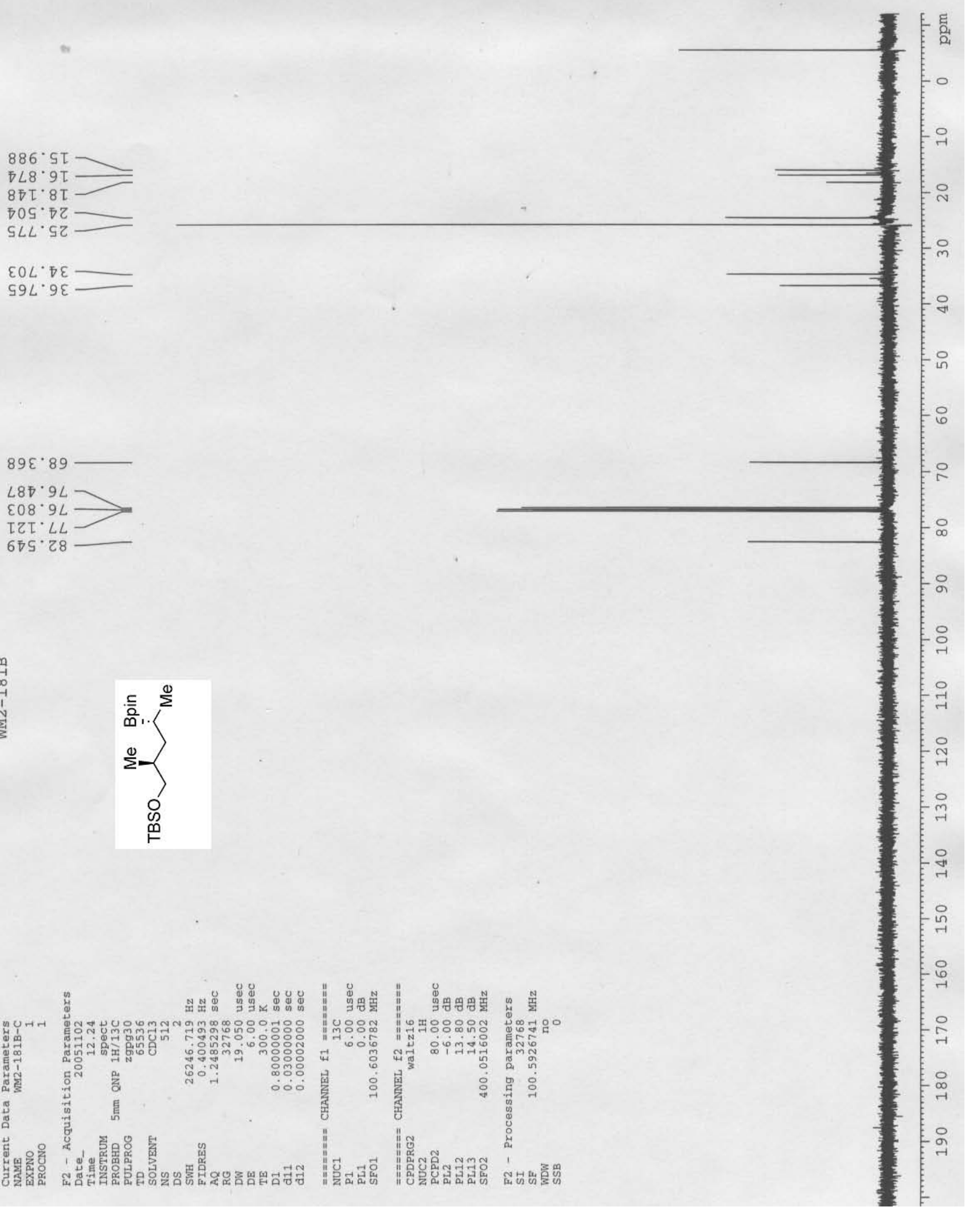
Page S-30

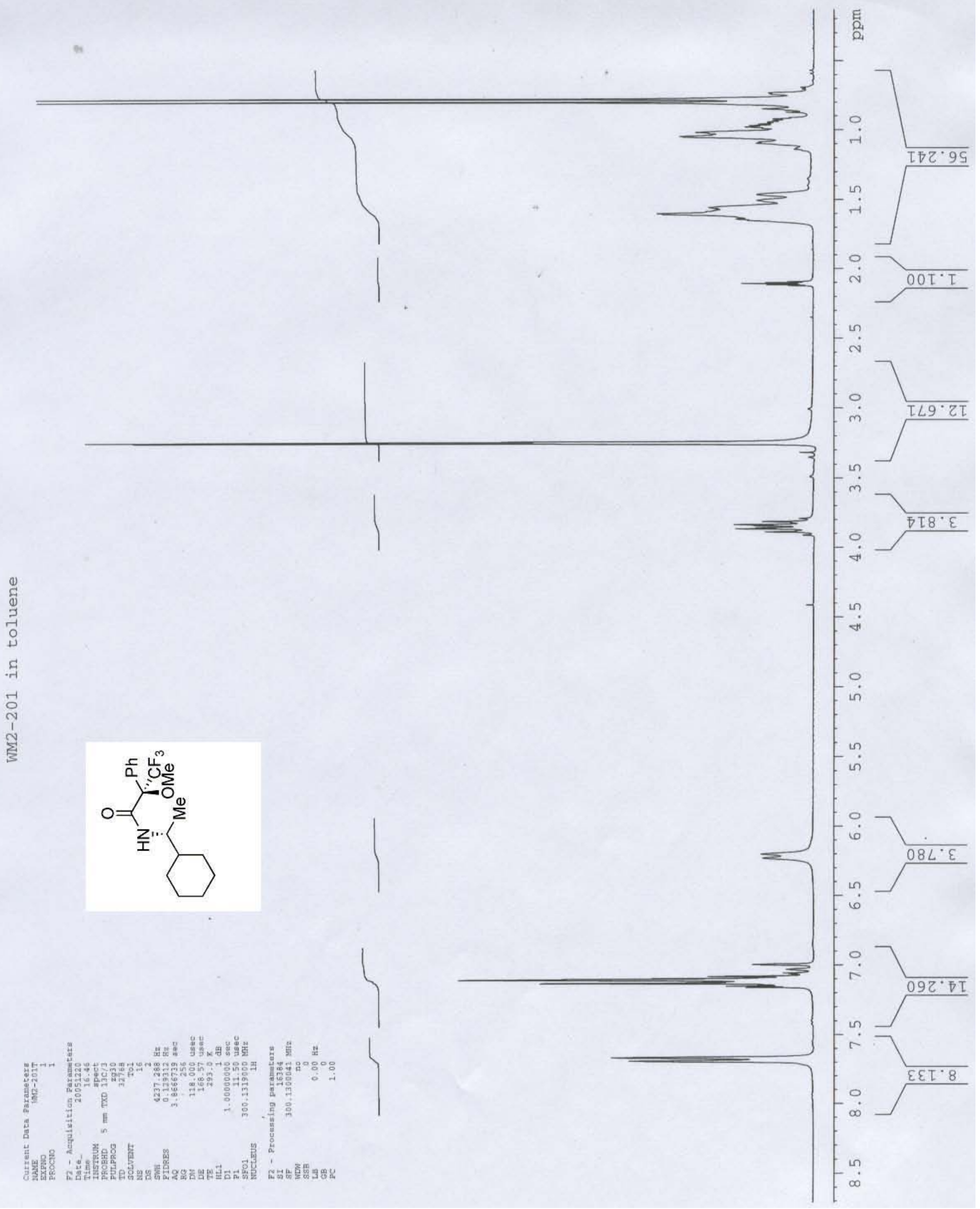


Page S-31

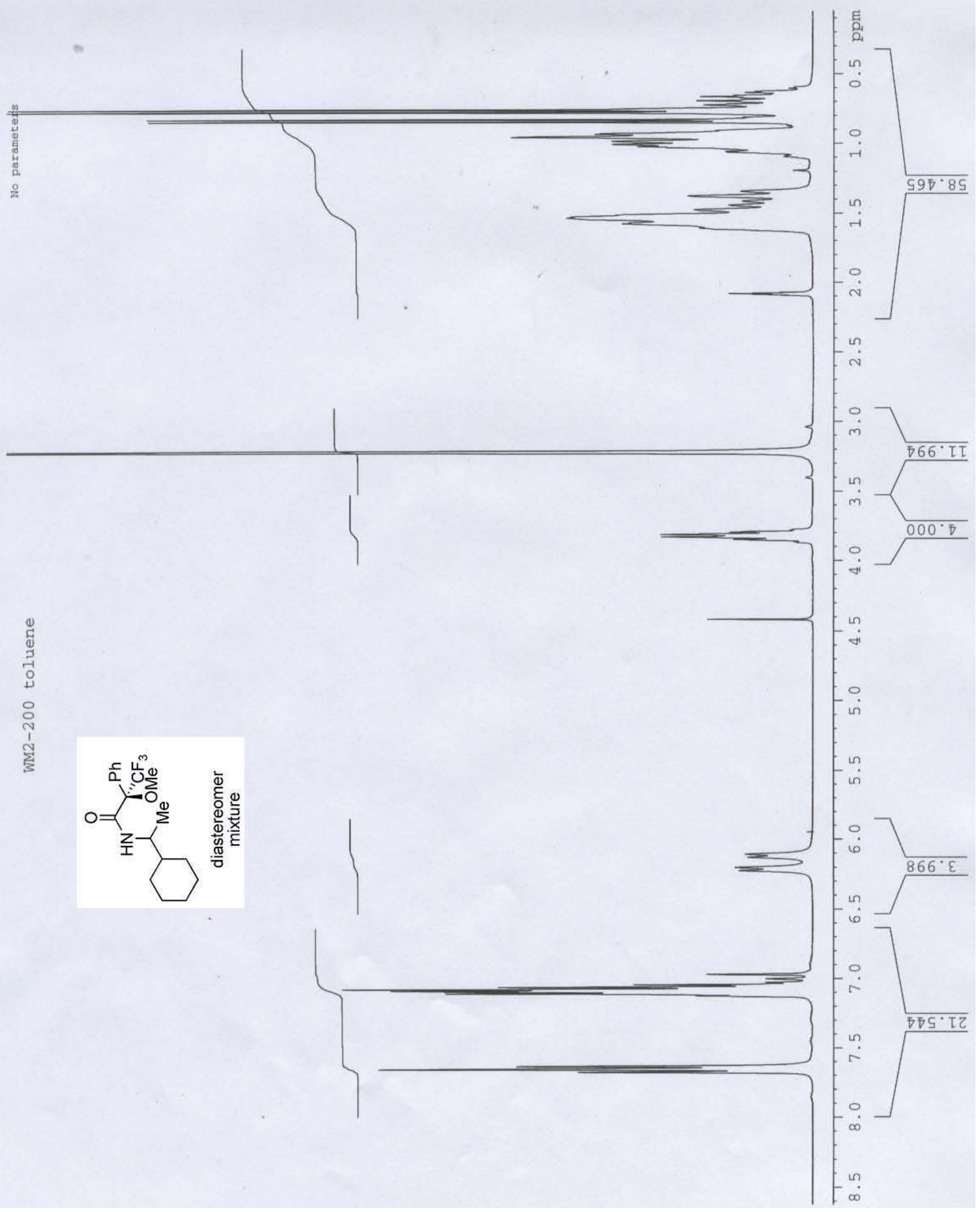

